Ingo Koller")/Florian Faust")

\title{
Die Haftung der Erstbank für Verschulden der Zwischenbank bei der Giroüberweisung
}

Im modernen Giroverkebr fübren Banken Überweisungsaufträge durchaus nicht immer durch einfaches Gutschreiben des Überweisungsbetrages auf einem Konto der Empfängerbank durch. Häufig werden von der Erstbank Zwischenbanken beauftragt, die ibrerseits den Betrag der Empfängerbank gutschreiben oder sogar weitere Zwischenbanken einschalten. Der vorliegende Beitrag will die Haftungsfragen beleuchten, die entstehen, wenn die Überweisung bei Einschaltung einer Zwischenbank nicht richtig weitergeleitet wird.

*) Dr. jur., Universitätsprofessor in Regensburg

$\because *)$ Cand.jur. in Regensburg
Inhaltsübersicht

I. Einführung

1. Problemstellung

2. Herkömmliche Lösungsansätze

3. Nachteile der herkömmlichen Lösungsansätze

II. Stand von Literatur und Rechtsprechung zur Haftung der Erstbank

1. Die Ansicht Köndgens

2. Die herrschende Meinung

3. Kritik der herrschenden Meinung 
3.1 Berufung auf die Rechtsprechung

3.2 Argument der fehlenden Erfolgsbezogenheit des Girovertrages

3.3 Argument der anderweitigen Kundenerwartungen

3.4 Argument der Unzumutbarkeit einer Haftung

III. Die Reichweite der Verpflichtung der Erstbank

IV. Die Haftungsbeschränkung analog $§ 664$ Abs. 1 Satz 2 BGB

1. Problem

2. Analoge Anwendung des $§ 664$ Abs. 1 Satz 2 BGB auf Geschäftsbesorgungsverträge

2.1 Stand von Rechtsprechung und Literatur

2.2 Ursprung des Problems

2.3 Ratio des $§ 664$ Abs. 1 Satz 2 BGB

2.4 Voraussetzungen einer Analogie

3. Anwendung des $§ 664$ Abs. 1 Satz 2 BGB bei der Einschaltung von Zwischenbanken

3.1 Handeln auf fremde Rechnung

3.2 Ermessensspielraum der Erstbank

3.3 Zwischenergebnis

4. Tatbestandsvoraussetzungen von $\S 664$ Abs. 1 Satz 2 BGB

4.1 Übertragung des Auftrags an einen Dritten

4.2 Gestattung der Substitution

5. Haftung für culpa in eligendo

V. Haftung im Licht der AGB-Banken

1. Nr.9 AGB-Banken

2. Nr.25 Abs. 1 AGB-Banken

VI. Zusammenfassung

\section{Einführung}

\section{Problemstellung}

Zwischenbetriebliche Überweisungen werden in der Regel nicht dadurch ausgeführt, daß die Erstbank den Betrag auf einem bei ihr geführten Konto der Empfängerbank gutschreibt oder daß diese ein bei ihr geführtes Konto der Erstbank belastet. Vielmehr beauftragt die Erstbank häufig eine Zwischenbank, die ihrerseits den Überweisungsbetrag der Empfängerbank gutschreibt oder eine weitere Zwischenbank einschaltet.

Wird die Überweisung durch eine Zwischenbank schuldhaft nicht richtig weitergeleitet, entsteht die Frage, an wen sich der Kunde der Erstbank halten kann. Ein vom OLG Düsseldorfentschiedener Fall ${ }^{1)}$ mag das verdeutlichen: Eine Zwischenbank gibt den Text eines bei ihr richtig eingegangenen Blitzgiros verstümmelt, nämlich ohne Namen und Kontonummer des Empfängers, an dessen Bank weiter. Diese verbucht daraufhin den Überweisungsbetrag zunächst auf dem Konto „pro Diverse“; die Gutschrift auf dem Empfängerkonto kann erst nach Ablauf des Kalenderjahres erfolgen. Weil der Empfänger eine Abschreibungsgesellschaft ist, verliert der Auftraggeber der Überweisung Steuervorteile.

\section{Herkömmliche Lösungsansätze}

Die herrschende Meinung eröffnet dem Geschädigten unmittelbar oder mittelbar einen Anspruch gegen die schuldhaft agie- rende Zwischenbank. Zum Teil wird mit der Figur der Drittschadensliquidation gearbeitet. ${ }^{2)}$ Diejenige Bank, die die Zwischenbank beauftragt habe, könne von dieser vertraglich den Schaden ihres Kunden ersetzt verlangen, da sie als mittelbare Stellvertreterin zur Drittschadensliquidation berechtigt sei, und werde gegenüber dem Kunden durch Abtretung dieses Schadensersatzanspruchs gemäß $§ 667$ BGB frei. Der Kunde kann demnach aus abgetretenem Recht gegen die Zwischenbank vorgehen.

Andere Autoren sehen die Verträge zwischen den mit der Überweisung befaßten Banken als Verträge mit Schutzwirkung für Dritte an, so daß der geschädigte Kunde der Erstbank, eventuell auch der Empfänger, unmittelbare Ansprüche gegen die schädigende Zwischenbank erheben kann. Dieser Lehre hat sich der BGH mehrfach bedient, um Bankkunden im Recht des Zahlungsverkehrs über die Kette der eingeschalteten Banken hinweg unmittelbare Ansprüche gegen die schädigende Bank zu eröffnen. ${ }^{3)}$

In der Literatur wird auch dafür plädiert, dem Kunden der Erstbank und dem Empfänger einen unmittelbaren deliktischen Anspruch gegen die schädigende Zwischenbank zu geben. ${ }^{4)} \mathrm{Mö}$ schel nimmt an, daß zwischen allen Beteiligten am System des bargeldlosen Zahlungsverkehrs, also sowohl zwischen dem Kunden der Erstbank und der Zwischenbank als auch zwischen dem Empfänger und der Zwischenbank, eine Art Netzvertrag bestehe. Es sei nämlich für alle Beteiligten ein selbstverständliches Faktum, „daß die Einzelverträge in ihrer wechselseitigen Aufeinanderbezogenheit ein System konstituieren mit dem einheitlichen Zweck der Zahlungsdurchführung“. Folglich gesteht er dem Kunden der Erstbank bzw. dem Empfänger einen unmittelbaren vertraglichen Anspruch gegen die schädigende Bank zu. ${ }^{5)}$

\section{Nachteile der herkömmlichen Lösungsansätze}

Alle diese Lösungen haben den Vorteil, daß sich die schädigende Zwischenbank nicht mit dem Argument aus der Affäre ziehen kann, ihr Vertragspartner, die Erstbank oder eine andere Zwischenbank, habe keinen Schaden erlitten. Für den Kunden der Erstbank haben sie jedoch den Nachteil, daß er die Angelegenheit nicht mit „seiner Bank“ klären kann, da diese in der Regel kein eigenes Verschulden trifft. Zwar ist sie ihm zur Auskunft über die Schadensentstehung verpflichtet, soweit sie selbst Informationen besitzt ( $\$ 666$ BGB); die Realisierung des Schadensersatzanspruches gegen die schädigende Bank kann jedoch erhebliche Probleme aufwerfen ${ }^{6}$. Die Erstbank wird, wenn ihre Beziehungen zum Kunden zerrüttet sind, kaum ein eigenes In-

1) OLG Düsseldorf, Urt. v. 11.2.1982, ZIP 1982, $428=$ WM 1982, 575.

2) BGH, Urt. v. 12.5.1958, BGHZ 27, 241, 247; BGH, Urt. v. 11.3.1976, WM 1976, 904, 907; Hadding, Drittschadensliquidation und „Schutzwirkung für Dritte" im bargeldlosen Zahlungsverkehr, in: Festschrift Werner, 1984, S. 165, $180 \mathrm{ff}$; Schlegelberger/Hefermehl, HGB, 5. Aufl., Anh. zu $§ 365$ Rz. 100

3) BGH, Urt. v. 28.2.1977, BGHZ69, 82, 86; BGH, Urt. v. 23.9.1985, BGHZ 96 . $9,17=$ ZIP 1985, 1469; sehr eingehend OLG Düsseldorf, Urt. v. 11.2.1982, ZIP 1982, 428, 429 f; Canaris, Bankvertragsrecht, 3. Bearb., 1988, Rz.395, 397.

4) ข. Bar, Verkehrspflichten, 1980, S. 157 ff, 204 ff; sehr vage auch Köndgen, Bankenhaftung - Strukturen und Tendenzen - Generalbericht, in: Köndgen, Neue Entwicklungen im Bankhaftungsrecht, 1987, S.133, 141.

5) Möschel, Dogmatische Strukturen des bargeldlosen Zahlungsverkehrs, AcP 186 (1986), S. 187, 211, 222.

6) Vgl. Köndgen (Fußn.4), S. 151. 
teresse an rascher Aufklärung des Schadensherganges haben. Sie kann sich als Geschäftsbesorgerin darauf beschränken, den Kunden über die Weitergabe der Überweisung an die von ihr beauftragte Zwischenbank zu informieren und ihm eventuelle Auskunftsansprüche gegen diese abzutreten ( $(667 \mathrm{BGB})$. Es ist dann Sache des Kunden, von dieser Zwischenbank Auskunft über den Stand des Geschäftes zu verlangen. Erhält er sie nicht, kann er vermuten, daß diese Zwischenbank die Schädigerin ist, und sie auf Schadensersatz verklagen. Im Prozeß kann sich dann jedoch herausstellen, daß die Beklagte die Überweisung an eine weitere Zwischenbank weitergegeben hat. Der Kunde kann nun mit dieser dasselbe Spiel von vorn beginnen.

Freilich ist hier ein Extrem gezeichnet; außerdem stehen dem Kunden auch Ersatzansprüche gegen Banken zu, die unzulängliche Auskünfte erteilen. Der Geschädigte soll jedoch auch dann seinen Schaden möglichst einfach liquidieren können, wenn die beteiligten Banken nicht ordnungsgemäß ihren Pflichten nachkommen. Ferner darf das Prozeßrisiko der Auskunftsprozesse nicht vernachlässigt werden, das der Kunde von Stufe zu Stufe neu läuft, ohne sich durch eine Streitverkündung ( $\$ 72 \mathrm{ZPO})$ absichern zu können.

Selbst wenn der Auftraggeber zuverlässig und zügig darüber informiert wird, welche Zwischenbank den Fehler begangen hat, und wenn im Hinblick auf die Drittschadensliquidation die Abtretungskette vor Eintritt der Verjährung in Ordnung ist, wird der Kunde gezwungen, am Geschäftssitz der Zwischenbank zu klagen. Er kann also nicht „seine“ Bank am Ort der Filiale verklagen, die er mit der Überweisung beauftragt hat (§§ 17, 21 ZPO, Nr.26 AGB-Banken).

Dazu kommt, daß der Kunde gegen die Erstbank im Rahmen des Kontokorrents Verrechnungsmöglichkeiten haben kann, während ihm diese gegenüber der Zwischenbank fehlen werden. Der Erstbank können jedoch gegenüber der von ihr eingeschalteten Zwischenbank und dieser gegenüber der drittbeauftragten Bank Verrechnungsmöglichkeiten zustehen, die der Kunde nicht für sich fruchtbar machen kann. Die beauftragende Erstund die Zwischenbank werden deshalb auch von einer Insolvenz der schädigenden Zwischenbank, abstrakt gesehen, nicht ganz so hart getroffen. Da die beauftragende Bank die nachgeschaltete Zwischenbank auch nach deren Solvenz aussuchen kann, spricht vieles dafür, den Kunden nicht auf den unter Umständen mühsamen Weg zu verweisen, selbst den Schadensersatzanspruch gegen die schädigende Zwischenbank durchzusetzen.

\section{Stand von Literatur und Rechtsprechung zur Haftung der Erstbank}

\section{Die Ansicht Köndgens}

Um die Nachteile der herkömmlichen Lösungsansätze zu vermeiden, hat Köndgen vorgeschlagen, dem Kunden einen Schadensersatzanspruch gegen die Erstbank zu eröffnen, indem die Zwischenbanken als deren Erfüllungsgehilfen qualifiziert werden. $^{7)}$

In den AGB-Banken sei nicht gesagt, ob die Bank lediglich verpflichtet sei, die Überweisung unverzüglich auf dem schnellsten
Weg weiterzugeben, oder ob sie selbst die Empfängerbank mit der Gutschrift beauftragen müsse. Diese Frage könne auch nicht anhand des Kundengesprächs am Bankschalter geklärt werden. Es komme daher darauf an, wie die Bank das Kundenverhalten nach Treu und Glauben verstehen müsse. Der Kunde erwarte nun, daß die Erstbank die Überweisung binnen angemessener Frist auf den Weg bringe und für eine Weiterleitung bis zur Empfängerbank Sorge trage. Er sehe in dem Vorgang der Überweisung eine einheitliche Dienstleistung der Erstbank. Dies gelte auch für Auslandsüberweisungen, weil er sich ja vielfach bewußt an ein Kreditinstitut mit Auslandserfahrungen wende. Die Bank müsse sich auf diese Kundenerwartungen redlicherweise einlassen. Sie dürfe sich ebensowenig wie ein Generalbauunternehmer oder ein Reiseveranstalter darauf berufen, daß sie allein die Überweisung durchzuführen nicht in der Lage sei. Vielmehr begründe der Überweisungsauftrag eine Leistungs- und Haftungseinheit, weil das Produkt „Überweisung zur Empfängerbank“ seiner Beschaffenheit nach oder doch zu vertretbaren Kosten nur arbeitsteilig erstellt werden könne.

Köndgen sieht das Problem, daß Regreßprozesse gegenüber den Zwischenbanken notwendig werden können. Er meint es dadurch zu entschärfen, daß er auf die Möglichkeiten der Anspruchsabtretung und der Pauschalierung der Regresse nach dem Modell der Teilungsabkommen in der Versicherungswirtschaft verweist. $^{8)}$

\section{Die herrschende Meinung}

Die These Köndgens stieß auf verbreitete Kritik. Man sah keinen Anlaß, von der - bis dato nicht näher begründeten - herrschenden Meinung abzugehen, derzufolge sich die Pflicht der Bank in der unverzüglichen Weiterleitung des Überweisungsauftrages erschöpft. ${ }^{9)}$ Die Zwischenbank könne nur dann als Erfüllungsgehilfin angesehen werden, wenn eine vertragliche Risikoübernahme oder eine gesetzliche Risikozuweisung vorliege. In dem auf Geschäftsbesorgung gerichteten bargeldlosen Zahlungsverkehr besteht Hüffer zufolge aber weder für das eine noch für das andere ein Anhaltspunkt. ${ }^{10)}$ Insbesondere fehle eine werkvertragstypische Erfolgsorientierung. ${ }^{11)}$ Schröter argumentiert, der Kunde wisse, daß die Erstbank zumindest die Empfängerbank mit der Gutschrift beauftragen müsse. Ob er davon ausgehe, daß die Überweisung eine einheitliche Dienstleistung der Bank sei, sei letztlich nicht feststellbar, jedenfalls wolle er der Erstbank keine unzumutbaren Haftungsrisiken auferlegen. Eine Parallele zum Reiseveranstalter- und Generalbauunternehmervertrag sei unzulässig, weil die Pauschalreise „vorfabriziert“ sei und die Pflicht des Reiseveranstalters in der Koordination liege. Der Generalbauunternehmer verpflichte sich zur Erstellung eines

7) Köndgen (Fußn. 4), S. $146 \mathrm{ff}$

8) Köndgen (Fußn.4), S. 151

9) BGH, Urt. v. 18.12.1951, BGHZ 4, 244, 248; Schlegelberger/Hefermehl (Fußn. 2), Anh. zu $\$ 365$ Rz.46; Hadding (Fußn. 2), S. 167; Koller, Grundstrukturen des Bankhaftungsrechts unter besonderer Berücksichtigung des Zahlungsverkehrs, in: Köndgen, Neue Entwicklungen im Bankhaftungsrecht, 1987, S.21, 23f; Canaris (Fußn.3), Rz.315, 329, 390; Schönle, Bank- und Börsenrecht, 2. Aufl., 1976, S. 356.

10) Hüffer, Die Haftung gegenüber dem ersten Auftraggeber im mehrgliedrigen Zahlungsverkehr, ZHR 151 (1987), S. 93, 95.

11) Hüffer, Haftungsfragen im Bankrecht, WM 1987, 641, 644 
bestimmten Werkes und suche sich die dazu geeigneten Subunternehmer. Gegen das Risiko eines Fehlverhaltens der Subunternehmer könne er sich durch die Stellung von Vertragserfüllungsgarantien sachgerecht absichern. Im Unterschied zum Werkvertragsrecht nehme die Bank jeden Überweisungsauftrag an, ohrie vorher nachzuprüfen, welche Zwischenbanken einzuschalten seien, und ohne sich gegen das Risiko eines Fehlverhaltens der Zwischenbank absichern zu können. Bankkunden würden sehr erstaunt sein, wenn die Bank eine Überweisung mit der Begründung nicht sofort ausführen würde, es müsse zuerst festgestellt werden, ob sie geeignete Zwischenbanken finden könne. ${ }^{12)}$

\section{Kritik der herrschenden Meinung}

Die Argumente, die die herrschende Meinung für eine Beschränkung der Pflicht der Erstbank auf die Weiterleitung der Überweisung anführt, vermögen letztlich nicht zu überzeugen.

\subsection{Berufung auf die Rechtsprechung}

Häufig beruft sich die herrschende Meinung ${ }^{13)}$ auf eine Entscheidung des Reichsgerichts aus dem Jahre 1922. ${ }^{14)}$ Diese bezieht sich jedoch auf ein Akkreditiv, und das Reichsgericht stellt ausdrücklich fest, „die schwierige und bisher nicht geklärte Frage, wann bei Bankgeschäften, bei denen auf derselben Seite zwei Banken beteiligt sind, die eine Bank als Erfüllungsgehilfin anzusehen ist", brauche nicht grundsätzlich erörtert zu werden.

1925 bejaht das Reichsgericht für einen Fall, bei dem die Reichsbank als Zwischenbank die verspätete Abwicklung einer zwischenbetrieblichen Überweisung verursachte, sogar ausdrücklich die Anwendung des $\$ 278$ BGB. ${ }^{15)}$ Die Erstbank habe die Pflicht, den Erfolg herbeizuführen, daß der Überweisungsbetrag zur Bank des Empfängers gelange und diesem dort gutgeschrieben werde. Da die Erstbank zur Realisierung dieses Erfolges die Wahl unter mehreren möglichen "Wegen" gehabt habe, könne man nicht schlechterdings sagen, daß ihre Vertragspflicht ihrem Kunden gegenüber mit Einschaltung der Reichsbank erfüllt gewesen sei. Etwas anderes gelte nur, wenn die Überweisung nur durch Hinzuziehung der Reichsbank hätte ausgeführt werden können und der Kunde mit dieser Tatsache rechnete oder rechnen mußte.

In der Entscheidung vom 7.4.1933 ${ }^{16)}$ ging es dem RG nicht um eine Frage des $§ 278$ BGB. Allerdings qualifizierte es den Überweisungsauftrag ohne nähere Begründung als Kommissionsgeschäft i.S.d. §406 Abs. 1 HGB. 1930 stellt erstmals das OLG Hamburg in einem obiter dictum fest, bei einer Giroüberweisung handle es sich um eine Aufgabe, die nicht „durch Erfüllungsgehilfen zu erledigen (sei), sondern durch eine Kette selbständiger Aufträge, die von einem Bankinstitut an ein anderes zu erteilen (seien) “. ${ }^{17)}$

Auch der BGH hat mehrfach angenommen, daß die Erstbank mit der Weiterleitung des Überweisungsauftrags an die Girozentrale und der Gutschrifterteilung zu deren Gunsten alles getan habe, was banküblicherweise von ihr zur Ausführung des Auftrags zu erwarten sei. ${ }^{18)}$ Näher begründet hat er diese Position jedoch nie. Insbesondere hat er nicht dem Umstand Rechnung getragen, daß sich die Pflichten der am Überweisungsverkehr
Beteiligten dadurch geändert haben könnten, daß die Giroüberweisung zum gängigen Zahlungsmittel der Privatkundschaft geworden ist.

\subsection{Argument der fehlenden Erfolgsbezogenheit des Girovertrages}

Hüffer führt aus, es fehle die für eine Haftung der Erstbank nach $\S 278$ BGB erforderliche vertragliche Risikoübernahme oder gesetzliche Risikozuweisung, weil die Bank bei der Giroüberweisung nur eine Dienstleistung und keinen Erfolg schulde. ${ }^{19)}$

In der Tat wird überwiegend angenommen, daß der Girovertrag ein Dienstvertrag sei. ${ }^{20)}$ Canaris begründet dies etwa damit, daß die Bank nicht immer wissen könne, ob der Überweisungsempfänger überhaupt ein Konto bei der Empfangsbank habe. ${ }^{21)}$ Mit demselben Argument müßte man dann aber den Frachtvertrag entgegen allgemeiner Ansicht als Dienstvertrag qualifizieren, weil auch beim Frachtvertrag nicht sicher ist, ob der Empfänger am Empfangsort anzutreffen ist und ob er das Gut übernimmt (vgl. nur §437 HGB). Wenn die Einordnung des Girovertrages als Dienstvertrag gleichwohl sachgerecht ist, so deshalb, weil der vereinbarte Entgeltmodus (Gebühren, Bodensatz, Wertstellung) nicht erfolgsorientiert ist.

Der Umstand, daß der Girovertrag als Dienstvertrag einzuordnen ist, steht jedoch der Anwendbarkeit des $§ 278$ BGB nicht entgegen. Andernfalls müßte z.B. ein Rechtsanwalt nie im Rahmen der Prozeßführung für seine Mitarbeiter einstehen. Entscheidend ist nach $\S 278$ BGB vielmehr allein der Umfang der übernommenen Pflicht, ob sich also die Bank zur bloßen Organisation (d.h. zur Weiterleitung an eine geeignete Zwischenbank) oder zur Durchführung der Überweisung verpflichtet hat.

Auch daß der Girovertrag kein „normaler" Dienstvertrag ist, sondern sich auf eine Geschäftsbesorgung richtet, erlaubt es nicht, von vornherein Zwischenbanken aus dem Kreis der Erfüllungsgehilfen nach $\S 278$ BGB herauszunehmen. Soweit das Geschäftsbesorgungselement Modifikationen erfordert, sind diese $\S 664$ BGB zu entnehmen und nicht in die Willenserklärungen der Parteien hineinzuinterpretieren.

\subsection{Argument der anderweitigen Kundenerwartungen}

Auch Schröters Argument, der Auftraggeber einer Überweisung gehe wohl nicht von einer einheitlichen Dienstleistung seines Kreditinstituts aus, da er wisse, daß es zumindest die Empfängerbank mit der Gutschrift beauftragen müsse, ${ }^{22)}$ ist nicht stichhaltig. Sicherlich wird auch aus Sicht der Kunden die Empfänger-

12) Schröter, Bankenhaftung im mehrgliedrigen Zahlungsverkehr, ZHR 151 (1987), S. 118, $120 \mathrm{ff}$.

13) Etwa Hüffer, ZHR 151 (1987), S. 93, 95, Fußn. 5.

14) RG, Urt. v. 17.6.1922, RGZ 105, 48, 51 = BankArch 1923, 261.

15) RG, Urt. v. 27.6.1925, LZ 1926, 45, 46.

16) RG, Urt. v. 7.4.1933, LZ 1933, 770, 771

17) OLG Hamburg, Urt. v. 16.6.1930, JW 1931, 548, 549; vgl. auch Anm. Ohse, JW 1931, 548, 549.

18) BGHZ 4, 244, 248.

19) Hüffer, WM 1987, 641, 644.

20) Schlegelberger/Hefermebl(Fußn.2), Anh. zu $§ 365$ Rz. 14 fm.w. N.; v. Gablenz, Die Haftung der Banken bei Einschaltung Dritter, 1983, S. 222.

21) Canaris (Fußn. 3), Rz. 315.

22) Schröter, ZHR 151 (1987), S. 118, $120 \mathrm{f}$. 
bank nicht mehr zum Risikobereich der Erstbank zählen, ${ }^{23)}$ weil sie davon ausgehen, letztere habe mit der Ablieferung des Geldes bei der Empfängerbank ihre Pflicht getan. In bezug auf die Zwischenbanken dürfen jedoch daraus keine Folgerungen gezogen werden.

\subsection{Argument der Unzumutbarkeit einer Haftung}

Das wichtigste Argument der herrschenden Meinung ist wohl, eine Haftung für die Zwischenbanken nach $§ 278$ BGB sei der Erstbank nicht zuzumuten, da sie Überweisungsaufträge wie selbstverständlich annehme, ohne sich zu vergewissern, ob geeignete Zwischenbanken zur Verfügung stünden, und da sie das Verhalten der in die Girokette eingeschalteten Institute nicht steuern und deren Auswahl nach der Weitergabe an die erste Zwischenbank nicht beeinflussen könne. ${ }^{24)}$ Näher belegt werden diese „unzumutbaren Haftungsrisiken“ allerdings nicht.

3.4.1 Das aus der fehlenden Steuerbarkeit der Zwischenbanken resultierende Risiko steht einer Haftung der Erstbank nach §278 BGB nicht entgegen. ${ }^{25)}$ Andernfalls könnte $§ 278$ BGB nie auf selbständige Erfüllungsgehilfen, §431 HGB nie auf eine Kette von Unterfrachtführern angewendet werden, weil der Umfang der Verpflichtung immer schon von vornherein so eingeengt werden müßte, daß selbständige, nicht wie Arbeitnehmer steuerbare Dritte gar nicht Erfüllungsgehilfen werden können. Vielmehr muß man der Wertung der $\S \S 278$ BGB, 431 HGB entnehmen, daß es bei der Lösung der Frage, wie weit der Rahmen der Verpflichtung des Schuldners zu ziehen ist, nicht primär auf die Selbständigkeit der vom Schuldner notwendig oder sinnvoll einzusetzenden Dritten ankommen kann. Zu berücksichtigen ist nur, daß der Schuldner, soweit ein anderweitiger subjektiver Verpflichtungswille nicht nachweisbar ist, nach Treu und Glauben nicht mit unerträglichen Haftungsrisiken konfrontiert werden darf.

3.4.2 Diesbezüglich kommt zunächst die Gefahr in Betracht, daß die Erstbank haften muß, wenn der Überweisungsbetrag wegen der Insolvenz oder eines Fehlverhaltens einer Zwischenbank „verlorengeht", nachdem ihn die Erstbank der ersten Zwischenbank gutgeschrieben hat. Häufig wird die Erstbank das Geld von der ersten Zwischenbank zurückerhalten können. Wenn dies nicht so ist, kann sie, falls ihr bei der Einschaltung der ersten Zwischenbank kein Verschulden zur Last fiel, grundsätzlich nach $\S 670$ BGB den Betrag vom Kunden ersetzt verlangen, auch wenn der angestrebte „Erfolg“ der Überweisung - die Gutschrift auf dem Empfängerkonto - aus irgendeinem Grund nicht eingetreten ist. Gleiches gilt, wenn eine Zwischenbank den Widerruf einer Überweisung schuldhaft unbeachtet läßt. Wendet man allerdings §278 BGB an, so kann der Kunde in der Regel wegen positiver Forderungsregelung mit einem Schadensersatzanspruch aufrechnen. Der Schaden resultiert daraus, daß die vom Kunden veranlaßten Aufwendungen wegen eines der Erstbank zuzurechnenden Verschuldens nicht den gewünschten Erfolg haben.

3.4.3 Es ergeben sich weitere Haftungsrisiken: daß eine Zwischenbank die Überweisung verspätet ausführt oder das Bankgeheimnis verletzt und dadurch einen Schaden verursacht. Letzteres wird sich nur selten realisieren, ersteres ist durch Nr.7 AGB-Banken weitgehend abbedungen - es fällt nur bei nich*- kaufmännischen Kunden und dann nur bei grobem Verschulden ins Gewicht. Zu beachten ist ferner, daß auch bei weiter Auslegung ihrer Verpflichtung die Erstbank nach $§ 664$ Abs. 1 Satz 2 BGB häufig von der Haftung für die Zwischenbanken befreit wird und daß die Fehlerquote bei Giroüberweisungen ohnehin verschwindend gering ist.

Selbst wenn die Erstbank zunächst für einen von einer Zwischenbank verursachten Schaden einstehen muß, muß sie ihn nicht notwendig auch im Ergebnis tragen, da sie gegen die von ihr beauftragte Zwischenbank oder nach den Regeln der Drittschadensliquidation bzw. des Vertrags mit Schutzwirkung für Dritte gegen die schädigende weitere Zwischenbank Regreß nehmen kann. Soweit diese solvent ist, macht der Regreß allenfalls unter dem Aspekt des Prozeßrisikos Schwierigkeiten. Dieses kann die Erstbank jedoch mit Hilfe des prozessualen Instruments der Streitverkündung ( $§ 72 \mathrm{ZPO})$ minimieren.

Es verbleibt damit die Gefahr, den Regreßanspruch wegen der Insolvenz der betreffenden Bank nicht realisieren zu können. Im Inlandsverkehr ist sie freilich äußerst gering, zumal der Erstbank häufig Verrechnungsmöglichkeiten zustehen werden. Anders mag es im grenzüberschreitenden Zahlungsverkehr sein. Geht man auch im Interbankenverhältnis davon aus, daß sich die Zwischenbank gegenüber der Erstbank nicht nur zur Weiterleitung, sondern auch zur Durchführung der Überweisung verpflichtet, kann die Erstbank ihrerseits die von ihr eingeschaltete erste Zwischenbank nach $\S 278$ BGB für das Fehlverhalten aller von dieser nachgeschalteten Institute haftbar machen; deren Solvenz kann ihr gleichgültig sein. Unter Banken ist jedoch allgemein bekannt, daß sich Zwischenbanken nur zur Weitergabe der Überweisung verpflichten wollen, so daß im Interbankenverhältnis eine Haftung nach $§ 278$ BGB wohl ausscheidet. Die Erstbank kann daher mit einem Regreß gegen eine ominöse „xyBank in Indien “26) konfrontiert sein, die sie selbst nicht ausgewählt hat, die möglicherweise sogar eingeschaltet werden mußte, weil sie in ihrem Staat ein Monopol für die Weitergabe von Überweisungen besitzt. Nur in dieser Fallgruppe erscheint die Annahme, das Haftungsrisiko werde durch eine „weite“ Auslegung der Verpflichtung der Erstbank unerträglich erhöht, nicht gänzlich unplausibel.

3.4.4 Hier stellt sich die Frage, ob die Erstbank solche besonderen Risiken auf sich nehmen muß oder ob sie sie vermeiden kann, indem sie entsprechende Überweisungsaufträge ablehnt. Der Überweisungsauftrag wird allgemein als (einseitige) Weisung im Rahmen eines Geschäftsbesorgungsverhältnisses qualifiziert, die bindend ist. ${ }^{27)}$ Dabei sind aber die Grenzen der Weisungsbefugnis zu berücksichtigen: unangemessene Weisungen darf der Kunde im Rahmen des Girovertrages nicht erteilen. Dazu gehören Überweisungsaufträge, die die Bank unangemes-

23) So auch Köndgen (Fußn. 4), S. $146 \mathrm{f}$.

24) Hüffer, WM 1987, 641, 644; Schröter, ZHR 151 (1987), S. 118, 121 f; Canaris (Fußn. 3), Rz. 390.

25) Vgl. Köndgen (Fußn.4), S. 150; Staudinger/Löwisch, BGB, 12. Aufl., §278 Rz. 11; Soergel/Wolf, BGB, 11. Aufl., §278 Rz.26; MünchKomm-Hanau, BGB, 2. Aufl., $278 \mathrm{Rz} .2$.

26) v. Gablenz (Fußn. 20), S. 205

27) Canaris (Fußn.3), Rz.320f; Meyer-Cording, Das Recht der Banküberweisung, 1951, S. 32 . 
sen belasten, weil sie über keine geeigneten Zwischenbanken verfügt, und die daher auch bei einer bloßen Weiterleitungspflicht der Erstbank zurückgewiesen werden müßten, und solche, die ihr unangemessene Risiken aufbürden. Derartige Weisungen sind als bloße Offerten zur Ausführung einer Überweisung zu behandeln, die die Bank, gegebenenfalls stillschweigend ( 362 HGB), annehmen oder auch ablehnen kann. Selbst wenn die Weisung im Einzelfall bindend ist, muß die Bank nicht notwendig Aufträge ausführen, die sie aus ihrer Sicht mit zu großen Haftungsrisiken belasten. Sie kann nämlich jederzeit den Girovertrag kündigen (Nr. 17 Satz 1 AGB-Banken), wenn der Kunde nicht auf ihr Angebot eingeht, ihre Pflicht bei dem entsprechenden Überweisungsauftrag auf die Weiterleitung zu beschränken.

Wie man auch ansetzt: die Bank kann immer verhindern, daß sie im Einzelfall in erkennbar unangemessene Haftungsrisiken hineingetrieben wird.

3.4.5 Dabei ist es rechtlich irrelevant, daß die Erstbank normalerweise Überweisungsaufträge entgegennimmt, ohne sich vorher über mögliche Zwischenbanken und eventuelle Haftungsrisiken zu informieren. Denn auch wenn sich die Banken üblicherweise zur Durchführung der Überweisung über alle Zwischenstationen hinweg verpflichten würden, würden sie Routineaufträge ohne nähere Prüfung entgegennehmen und erledigen. Umgekehrt dürfte bei einer bloßen Weiterleitungspflicht die Erstbank die Aufträge nicht einfach bestmöglich ausführen, sondern müßte den Kunden, der in diesem Fall das Risiko zu tragen hätte, über die zweifelhafte Solvenz einzuschaltender Zwischenbanken und die schwere Realisierung etwaiger Schadensersatzansprüche gegen diese aufklären, um ihm Gelegenheit zu geben, einen anderen Überweisungsweg zu wählen. Unabhängig von der Reichweite ihrer Verpflichtung muß die Erstbank daher vor der Ausführung „zweifelhafter" Überweisungen mögliche Risiken prüfen, wenn sie sich nicht schadensersatzpflichtig machen will.

3.4.6 Die Behauptung, mit einer „weiten“ Auslegung ihrer Verpflichtung würden der Bank unerträgliche Haftungsrisiken aufgebürdet, vermag daher ebensowenig zu überzeugen wie die anderen Argumente der herrschenden Meinung.

\section{Die Reichweite der Verpflichtung der Erstbank}

Maßgeblich für das der Bank dem Kunden gegenüber obliegende Pflichtenprogramm ist der Girovertrag, der wiederum im wesentlichen durch die AGB-Banken „ausgefüllt" wird. Nach diesen steht „die Bank ihren Kunden mit ihren Geschäftseinrichtungen zur Erledigung verschiedenartigster Aufträge zur Verfügung“. Dazu gehören auch Überweisungsaufträge, die in Nr.7 Abs. 3 AGB-Banken ausdrücklich genannt werden und für deren Erteilung die Bank spezielle Formulare bereithält.

Die Bank verpflichtet sich also schon im Girovertrag, für ihre Kunden grundsätzlich Überweisungsaufträge auszuführen. Der einzelne Überweisungsauftrag ist eine einseitige Weisung i. S.d. $\S \S 675,665 \mathrm{BGB},{ }^{28)}$ durch die die girovertragliche Pflicht der Bank in Hinblick auf den Einzelfall konkretisiert wird (bezüglich des Zeitpunkts der Ausführung, des Empfängers und des
Betrages) - die Leistung „Überweisung“ an sich ist aber schon durch den Girovertrag vorgegeben.

Ob die Bank generell Durchführung oder bloße Weiterleitung der Überweisung schuldet, muß daher durch Auslegung des Girovertrages nach $\S 157$ BGB ermittelt werden. Da es um die Auslegung von $A G B$ geht, ist auf den objektiven Inhalt und den typischen Sinn der Formulierung „Überweisungsauftrag“ abzustellen, auf die Verständnismöglichkeiten des Durchschnittskunden ohne bankrechtliche Spezialkenntnisse. Im Rahmen dieses Verständnishorizontes ist die Formulierung so auszulegen, wie sie verständige und redliche Partner unter Abwägung der Interessen der normalerweise beteiligten Kreise verstehen müssen. Dies gilt auch für die konkrete Weisung, die in der Regel mit Hilfe eines Formulars erteilt wird, in dem nurvon „Überweisungsauftrag an X-Bank" die Rede ist.

Bislang sind empirische Untersuchungen darüber, wie Durchschnittskunden die Formulierung „Überweisungsauftrag“ verstehen, nicht angestellt worden. Im Falle eines Prozesses müßten derartige Erhebungen, sei es auch nur durch eine Umfrage der Industrie- und Handelskammer unter Kaufleuten, dringend nachgeholt werden. Soweit die Verfasser Erhebungen gemacht haben, die sicherlich nicht den Anforderungen an Umfragen genügten, waren die befragten Bankkunden ganz selbstverständlich der Meinung, daß die Geldüberweisung wie der Transport von Geld in völliger Verantwortung der Erstbank abzuwickeln sei. Mit den Zwischenbanken habe man nichts zu tun, war die stereotype Antwort.

Diese Laien-Erwartung ist auch im nicht-juristischen Schrifttum verbreitet. So definiert das Wörterbuch von Brockhaus und Wahrig ${ }^{29)}$ „Überweisungsauftrag“ als „Auftrag des Kontoinhabers an seine Bank, zu Lasten seines Kontos einen bestimmten Betrag auf ein anderes Konto bei der gleichen oder einer anderen Bank zu übertragen“. Bei Meyer ${ }^{30)}$ heißt es: Überweisung ist ein „Geschäftsbesorgungsvertrag eines Kontoinhabers mit einem Kreditinstitut, wodurch dieses verpflichtet wird, zu Lasten des Girokontos des Kontoinhabers einen bestimmten Betrag dem Konto des Zahlungsempfängers gutzuschreiben bzw. bei der Bank des Empfängers gutschreiben zu lassen“. Selbst das „Enzyklopädische Lexikon für das Geld-, Bank- und Börsenwesen“311 führt aus: „Durch die Überweisung wird der Zahlungsbetrag dem Girokonto des Zahlungspflichtigen belastet und dem Konto des Zahlungsempfängers gutgeschrieben“.

Mit „Überweisung“ wird demnach im allgemeinen Sprachgebrauch die Tätigkeit bezeichnet, die dazu führt, daß Buchgeld bei der Empfängerbank ankommt, möglicherweise sogar der Erfolg der Ankunft. Daß diese Deutung im Laien-Bewußtsein verankert ist, ist nicht sehr verwunderlich, wenn man bedenkt, daß aus der Sicht des Durchschnittskunden die Bank mit einer Tätigkeit betraut wird, die sich in einem mit höchster Wahrscheinlichkeit zu erreichenden Erfolg niederschlagen soll. In solchen Fällen wird der Auftragnehmer üblicherweise nicht lediglich mit der Einschaltung anderer Unternehmen beauftragt;
28) Siehe oben Fußn. 27
29) Brockhaus/Wahrig, Deutsches Wörterbuch, 1984
30) Meyers Enzyklopädisches Lexikon, Band 24, 1979
31) 3.Aufl., Frankfurt, $1967 / 68$ 
der Laie denkt ja auch nicht daran, den Maler mit der Einschaltung anderer Unternehmen zu beauftragen, wenn er ihm den Auftrag zum Streichen des Hauses gibt und der Maler Subunternehmer einschaltet. Diese dem Auftragnehmer erkennbare Erwartung des Auftraggebers muß der Auftragnehmer grundsätzlich respektieren, wenn er darauf verzichtet, die Erwartung zu korrigieren.

Bedenken gegen eine derartige am Verständnishorizont der Allgemeinheit orientierte Auslegung wären allerdings angebracht, wenn durch sie der Bank unzumutbare Belastungen auferlegt würden. Wie oben dargetan wurde, ist dies jedenfalls typischerweise im innerdeutschen Zahlungsverkehr nicht der Fall. Im grenzüberschreitenden Zahlungsverkehr mag eine unverhältnismäßig hohe Belastung in Einzelfällen zu bejahen sein. Ob sie auch als Charakteristikum des grenzüberschreitenden Zahlungsverkehrs angesehen werden kann, erscheint fraglich. In Zweifelsfällen kann auf das Auslegungsinstrument der Unklarheitenregel zurückgegriffen werden. Die sich aus dem Girovertrag ergebenden Rechtsbeziehungen zwischen Kunde und Bank werden durch die AGB-Banken geregelt. Der darin und in den Formularen ${ }^{32)}$ enthaltene Begriff des Überweisungsauftrags ist daher gemäß $§ 5$ AGBG im Zweifel zugunsten des Kunden der Erstbank so auszulegen, daß die Bank nicht nur die Organisation, sondern auch die Durchführung der Überweisung schuldet.

Bei dieser Auslegung, die die Pflicht zur Durchführung von Überweisungsaufträgen schon aus dem Girovertrag ableitet, kommt es auf die Art der Erteilung des einzelnen Überweisungsauftrags nicht mehr an: ob der Kunde das bankübliche Überweisungsformular benutzt oder die Bank privatschriftlich oder telefonisch zum Überweisen auffordert, immer bezieht er sich auf die der Bank schon aus dem Girovertrag obliegende Leistung „Überweisung“, die nicht in der bloßen Weiterleitung, sondern in der Durchführung des Überweisungsauftrags besteht. Die Bank schuldet also dem Auftraggeber einer Überweisung immer dann den „Transport“ des Geldes bis zur Empfängerbank, wenn im Einzelfall keine Anhaltspunkte (wie z.B. die Vorgabe eines bestimmten „Überweisungsweges“ durch den Kunden) für eine Beschränkung ihrer Pflicht sprechen.

\section{Die Haftungsbeschränkung analog $\$ 664$ Abs. 1 Satz 2 BGB}

\section{Problem}

Erstreckt man im Einklang mit den typischen Kundenerwartungen die Vertragspflichten der Erstbank auf den gesamten Prozeß der Giroüberweisung bis zur Beauftragung der Empfangsbank, so hat dies nicht notwendig zur Konsequenz, daß die Erstbank für die Zwischenbank gemäß §278 BGB haften muß. Es ist nämlich denkbar, daß sie sich auf das Haftungsprivileg des $§ 664$ Abs. 1 Satz 2 BGB berufen kann.

$\S 675$ BGB erklärt eine Reihe von auftragsrechtlichen Vorschriften für auf entgeltliche Geschäftsbesorgungsverträge entsprechend anwendbar, $\S 664$ BGB befindet sich allerdings nicht darunter. Das Haftungsprivileg des $\S 664$ Abs. 1 Satz 2 BGB kann deshalb der Erstbank nur dann zugute kommen, wenn der
Nachweis gelingt, daß die Beschränkung der Haftung auf culpa in eligendo auch im Rahmen entgeltlicher Geschäftsbesorgungsverträge ( $\$ 675$ BGB) zum Tragen kommt. Kann dieser Nachweis geführt werden, muß die Erstbank darüber hinaus beweisen, daß die Zwischenbank nicht lediglich ihr Gehilfe i. S.d. $\S 664$ Abs. 1 Satz 3 BGB war und daß ihr die Substitution gestattet war $(\S \S 613,664$ Abs. 1 Satz 1 BGB).

\section{Analoge Anwendung des $\S 664$ Abs. 1 Satz 2 BGB auf Geschäftsbesorgungsverträge}

\subsection{Stand von Rechtsprechung und Literatur}

Das RG führte in einer Entscheidung im 78. Band ${ }^{33)}$ aus, aus der Tatsache, daß $\S 675$ BGB gerade nicht auf $\S 664$ BGB verweise, dürfe kein Umkehrschluß gezogen werden. Aus der „Natur der Sache" heraus sei $§ 664$ Abs. 1 Satz 2 BGB auch aufDienst- oder Werkverträge mit Geschäftsbesorgungscharakter anzuwenden, wenn der Auftrag einem Dritten übertragen werde. Mit dieser Rechtsprechung hat das RG im 161. Band gebrochen. ${ }^{34)} § 613$ BGB wiederhole für den Dienstvertrag inhaltlich die Vorschriften von $\S 664$ Abs. 1 Satz 1 und Abs. 2 BGB, ein dem $\S 664$ Abs. 1 Satz 2 BGB entsprechendes Haftungsprivileg sei im Dienstvertragsrecht nicht vorgesehen. Für Dienst-und Werkverträge mit Geschäftsbesorgungscharakter gelte $\S 675$ BGB, der $§ 664$ BGB gerade nicht für anwendbar erkläre. Es bleibe daher bei der Haftung nach $§ 278$ BGB. Im 163. Band ging es um die Bestellung eines anwaltlichen Vertreters. ${ }^{35)}$ Das RG nahm zur analogen Anwendbarkeit des $\S 664$ Abs. 1 Satz 2 BGB nicht Stellung. Es bejahte jedenfalls im Fall der anwaltlichen Gesamtvertretung eine Haftung gemäß §278 BGB, weil der Mandant regelmäßig darauf vertraue, daß der von ihm gewählte Anwalt ihm gegenüber die Verantwortung übernehme.

Der BGH befürwortete $1951^{36)}$ unter Berufung auf das Reichsgericht ${ }^{37)}$ und ohne eigene Begründung eine analoge Anwendung von $\S 664$ BGB. Es läßt sich somit festhalten, daß die Rechtsprechung zur analogen Anwendbarkeit des §664 BGB noch nicht gefestigt ist.

Eine Reihe von Autoren lehnt die Anwendbarkeit des $§ 664$ Abs. $1 \mathrm{BGB}$ auf entgeltliche Geschäftsbesorgungsverträge generell ab. ${ }^{38)}$ Bezüglich $\S 664$ Abs. 1 Satz 1 und Abs. 2 BGB gelangen sie jedoch teilweise zum gleichen Ergebnis, indem sie auf $\S 613$ BGB verweisen oder im Fall von Werkverträgen ein Substitutionsverbot aus dem Vertrauensverhältnis ableiten. Der wohl überwiegende Teil der Literatur plädiert wegen der Rechtsähnlichkeit zwischen Auftrag und entgeltlicher Geschäftsbesor-

32) Vgl. BGH, Urt. v. 5.5.1986, ZIP 1986, $1042=$ BB 1986, 1462.

33) RG, Urt. v. 2.3.1912, RGZ 78, 310, 313.

34) RG, Urt. v. 4.7.1939, RGZ 161, 68, 70.

35) RG, Urt. v. 19.4.1940, RGZ 163, 377

36) BGH, Urt. v. 14.11.1951, LM BGB §664 Nr.1.

37) In dem in NJW 1952, 257 abgedruckten Leitsatz berief sich der BGH auf RGZ 161,68, das gerade entgegengesetzt entschieden hatte. Auf einen sehr kritischen Artikel von $v$. Bernstorff (Keine entsprechende Anwendbarkeit des $§ 664$ BGB auf entgeltliche Geschäftsbesorgungsverträge!, NJW 1952, 731) hin wurde in LM ein berichtigtes Blatt eingefügt, das auf RGZ 78, 310 verweist.

38) Erman/Hauß, BGB, 7. Aufl., §664 Rz.7; MünchKomm-Hanau, (Fußn. 25), \$278 Rz. 16; Steffen, in: BGB-RGRK, 12. Aufl., §664 Rz. 12; v. Bernslorff. NJW 1952, 731, 732; Metzler, Zur Substitution, insbesondere zu ihrer Abgrenzung von der Erfüllungsgehilfenschaft, AcP 159 (1960), S. 143, 154. 
gung, die in der Wahrnehmung fremder Vermögensinteressen besteht, für eine entsprechende Anwendbarkeit des $§ 664$ Abs. 1 BGB. ${ }^{39)}$

\subsection{Ursprung des Problems}

Nach den Vorstellungen der 1. Kommission zum Entwurf eines Bürgerlichen Gesetzbuches sollten die Vorschriften über den Auftrag sowohl die unentgeltliche als auch die entgeltliche Geschäftsbesorgung erfassen $(\$ \S 585,586 \mathrm{E} \mathrm{I})$, die Abgrenzung zum Dienst- und Werkvertrag wurde „Wissenschaft und Praxis“ überlassen. ${ }^{40)}$ Unabhängig von der Vereinbarung eines Entgelts untersagte der Entwurf im Zweifel die Einschaltung eines Substituten, weil die meisten Aufträge „nicht ohne Rücksicht auf persönliches Vertrauen ertheilt" würden. ${ }^{41)}$ Gestatte der Auftraggeber die Substitution, so liege in ihr in der Regel die Erfüllung der aus dem Auftrag entspringenden Verpflichtung des Beauftragten. ${ }^{42)}$ Dabei verstand die 1 . Kommission unter Substituten Dritte, denen das Geschäft zur „selbständigen Ausübung“ überlassen werde. ${ }^{43)}$ Keine Substituten seien „Gehülfen“, bei denen der Beauftragte die Erledigung des Geschäfts selbst in der Hand behalte. ${ }^{44)}$ Die 2. Kommission hielt an der Abgrenzung zwischen Substitution und Einschaltung von "Gehülfen“ fest. Erstere sei, auch wenn nur ein Teil des Auftrags übertragen werde, im Zweifel verboten und gehe, wenn der Auftraggeber sie gestattet habe, auf dessen Gefahr. Letztere sei dagegen generell zulässig, entbinde aber den Beauftragten nicht von der Haftung nach §278 BGB. ${ }^{45)}$ Im Unterschied zur 1. Kommission erhob die 2. Kommission die Unentgeltlichkeit zum Charakteristikum des Auftrags ( $§ 593$ E II), die entgeltliche Geschäftsbesorgung sei abschließend durch die Vorschriften des Dienst- und Werkvertragsrechts geregelt. ${ }^{46)} \mathrm{Zu}$ deren Ergänzung wurde der Vorläufer des heutigen $\S 675$ BGB geschaffen, der außer auf die Kündigungs- bzw. Widerrufsregelung und den heutigen $§ 664$ BGB auf alle Normen des Auftragsrechts verweist. Warum gerade $\S 664$ BGB ausgenommen wurde, hat die 2. Kommission nicht begründet. ${ }^{47)}$

In dieser Situation läßt sich entgegen $H \ddot{u ̈ f f e}{ }^{48)}$ die Tragweite des $\S 664$ Abs. 1 Satz 2 BGB nicht durch einen simplen Rückgriff auf die in der 1. und 2. Kommission vertretene Ansicht ${ }^{49)}$ begründen, daß mit einer erlaubten Substitution die Verpflichtung des Auftragnehmers erfüllt sei und es damit an der von $§ 278 \mathrm{BGB}$ tatbestandlich vorausgesetzten Verbindlichkeit fehle. Denn dies ist lediglich die dogmatische Einordnung eines Ergebnisses; das eigentliche Problem stellt sich auf der darunterliegenden Stufe: Entscheidend ist, warum der Gesetzgeber annahm, daß im Auftragsrecht erlaubte Substitution Erfüllung bedeutet. Auf dieser Stufe muß nun geklärt werden, ob die Interessenlage bei unentgeltlichem Auftrag und entgeltlicher Geschäftsbesorgung im Licht der Wertungen des Gesetzgebers so ähnlich ist, daß eine analoge Anwendung des $§ 664$ BGB auf entgeltliche Geschäftsbesorgungsverträge möglich oder zur Vermeidung von Wertungswidersprüchen sogar geboten ist.

Allein auf diese Weise können auch Wertungswidersprüche zum normalen Dienstvertragsrecht und zum Depotrecht vermieden werden. Das RG hatte im 161. Band zu Recht darauf hingewiesen, daß das BGB im Dienstvertragsrecht ebenfalls im Zweifel die Substitution verbietet ( $\$ 613$ BGB), aber aus ihrer Erlaubnis gleichwohl nicht die Folgerung zieht, die dienstvertraglichen Pflichten seien durch sie erfüllbar. ${ }^{50)}$ Im Rahmen des $\S 3$ Abs. 2 Depot $G$ wird sogar ausdrücklich gesagt, daß der Beauftragte im Fall der Substitution für Verschulden des Substituten wie für eigenes Verschulden hafte. Zur Begründung einer analogen Anwendung des $\S 664$ Abs. 1 Satz 2 BGB im Rahmen des $\S 675$ BGB muß deshalb - um in den Kategorien Hüffers ${ }^{51)}$ zu sprechen - ermittelt werden, warum in $\$ 664$ BGB klargestellt worden ist, daß die Erlaubnis zur Substitution die Reichweite der Verpflichtung des Beauftragten beschränkt, und warum die gleiche Beschränkung, anders als bei einfachen Dienstverträgen, bei allen Dienstverträgen mit Geschäftsbesorgungscharakter Platz greifen soll. Es ist zu klären, warum der Arzt für seinen Substituten gemäß $§ 278$ BGB haften soll, der entgeltlich tätige Treuhänder, der überschaubare Risiken gut einkalkulieren kann, sich aber auf die Einschaltung eines Dritten zurückziehen darf, wenn beiden die Substitution erlaubt worden ist.

\subsection{Ratio des $§ 664$ Abs. 1 Satz 2 BGB}

2.3.1 Vereinzelt wird angenommen, der Grund für das Haftungsprivileg des $\$ 664$ Abs. 1 Satz 2 BGB liege in der Gestattung der Substitution durch den Auftraggeber. ${ }^{52)}$ Dieser habe mit der Erlaubnis zur Substitution zu erkennen gegeben, daß seiner Ansicht nach ein sorgfältig ausgewählter Substitut den Auftrag ebenso gut wie der Beauftragte erledigen werde und daß er deshalb auf dessen Haftung nicht mehr angewiesen sei.

Mit dem realen Parteiwillen des Auftraggebers läßt sich dieses Argument jedoch nur schlecht vereinbaren; denn welchen Anlaß sollte er dafür haben, wenn er, möglicherweise im Interesse des Auftragnehmers, die Substitution erlaubt, auch das Risiko in Kauf zu nehmen, bei einem ihm unbekannten Substituten Regreß nehmen zu müssen. Das RG führte daher im 163. Band mit guten Gründen aus, daß der Mandant regelmäßig darauf baue, daß der von ihm gewählte Anwalt auch im Fall einer Substitution weiter die Verantwortung für eine ordnungsgemäße Behandlung der Rechtssache trage. ${ }^{53)}$

Außerdem vermag diese Ansicht nicht zu begründen, warum die Gestattung der Substitution gerade im Auftrags-, nicht aber im Dienstvertrags-und Depotrecht zu einem Haftungsprivileg

39) MünchKomm-Seiler (Fußn. 25), §675 Rz. 5; Soergel/Mühl (Fußn. 25), §664 Rz. 4; Staudinger/Wittmann (Fußn. 25), §664 Rz. 13; ). Gablenz (Fußn. 20), S. 92 Kümpel, Zur Anpassung der AGB der Banken an das AGB-Gesetz, WM 1977 , 694, 700; Schröter, ZHR 151 (1987), S. 118, 124

40) Mot II, S. 526, 531.

41) Mot II, S. 531

42) Mot II, S. 532, 533.

43) Mot II, S. 532.

44) Mot II, S. 533

45) Prot II, S. $354 \mathrm{f}$.

46) Prot II, S. 352

47) v. Gablenz (Fußn. 20), S. 85, hält einen Irrtum des Gesetzgebers für wahrscheinlich.

48) Hüffer, WM 1987, 641, 643

49) Mot II, S.532; ebenso RG, Urt. v. 2.3.1912, RGZ 78, 310, 312

50) RG, Urt. v. 4.7.1939, RGZ 161, 68, 70

51) Hüffer, WM 1987, 641, 643.

52) Staudinger/Wittmann (Fußn. 25), §664 Rz.4.

53) RG, Urt. v. 19.4.1940, RGZ 163, 377. 
für den eigentlichen Schuldner führt. In Hinblick auf einen konkludenten Verzicht auf die weitere Verantwortung des Vertragspartners gibt es keine Unterschiede zwischen der Substitution beim Arztvertrag und der Substitution beim Treuhandvertrag. In beiden Fällen wird der Gläubiger (Patient bzw. Treugeber) seine Rechte nur insoweit aufgeben wollen, als dies mit der Einschaltung eines Substituten notwendigerweise unabdingbar verbunden ist. Er wird also in der Regel ausschließlich darauf verzichten wollen, daß gerade sein Vertragspartner aktiv wird, nicht aber, daß dieser haftet. ${ }^{54)}$

2.3.2 $\S 613$ BGB unterscheidet sich darin von $\S 664$ Abs. 1 Satz $1 \mathrm{BGB}$, daß er auf entgeltliche Dienstverträge bezogen ist. Es liegt daher nahe, anzunehmen, daß der Auftraggeber den Auftragnehmer deshalb mit der Erlaubnis zur Substitution aus dem Vertrag entlasse, weil der Beauftragte unentgeltlich tätig werde. In den Gesetzesmaterialien findet sich hierfür freilich kein Anhaltspunkt. An keiner Stelle wird die Unentgeltlichkeit des Auftrags als erheblich für die Substitutionsregelung angesehen. Im Gegenteil war schon in $\S 589$ des ersten Entwurfs zum $B G B$, der noch von einer möglichen Entgeltlichkeit des Auftrags ausging ( $\$ 586 \mathrm{EI}$ ), eine dem heutigen $\S 664 \mathrm{Abs}$. 1 Satz 2, 3 BGB entsprechende Regelung enthalten, und auch als im zweiten Entwurf die Unentgeltlichkeit zum Charakteristikum des Auftrags erhoben wurde, hielt die Kommission in bezug auf die Beziehungen zwischen einer juristischen Person und deren Vorstand, der im Normalfall nicht unentgeltlich arbeite, ${ }^{55)}$ die Verweisung auf die Substitutionsregelung ( $§ 44$ Abs. 2 E I, $§ 26$ Abs. 3 E II, §27 Abs. 3 BGB) ausdrücklich aufrecht. Der Gesetzgeber sah somit die Substitutionsregelung als mit einer entgeltlichen Geschäftsbesorgung durchaus vereinbar an. ${ }^{56)}$

2.3.3 Bei der Bestimmung der ratio von $\S 664$ Abs. 1 Satz 2 BGB muß berücksichtigt werden, daß die $\S \S 662$ ff BGB zwei heterogene Arten von Pflichten einheitlich regeln: die unentgeltliche Geschäftsbesorgung und die unentgeltliche Erbringung von Diensten ohne Geschäftsbesorgungscharakter bzw. die unentgeltliche Herbeiführung eines Erfolges. Soweit im konkreten Fall der Auftragnehmer keine Geschäftsbesorgungspflichten übernommen hat, sondern nur eine sonstige Tätigkeit bzw. die Herbeiführung eines Erfolges versprochen hat, ist die Rechtsfolge des $\S 664$ Abs. 1 Satz 2 BGB nur durch die Unentgeltlichkeit zu erklären. Allein sie rechtfertigt es, daß sich der kostenlos tätige Arzt, nicht aber der gegen Vergütung tätige Arzt, der kostenlos arbeitende Maler, nicht aber der gegen Entgelt arbeitende Maler auf $\S 664$ Abs. 1 Satz 2 BGB berufen darf, wenn er einen Substituten beauftragt. ${ }^{57)}$

Anders ist die Sachlage bei Geschäftsbesorgungspflichten. Sie sind auch bei entgeltlichen Geschäftsbesorgungsverträgen dadurch gekennzeichnet, daß sie dem Geschäftsbesorger typischerweise einen mehr oder minder großen Spielraum eröffnen, wie er die wirtschaftlichen Interessen des Auftraggebers fördern kann. Dieser Spielraum tritt besonders deutlich bei den gesetzlich normierten Sonderformen der Geschäftsbesorgung, der Kommission und der Spedition, hervor.

Von der Existenz eines wirtschaftlichen Ermessenspielraums her ist auch die Regelung des $\S 670$ BGB zu begreifen, die, wie $\S 675$ $\mathrm{BGB}$ ausdrücklich sagt, auch im Recht der entgeltlichen Ge- schäftsbesorgung gilt. Der Geschäftsbesorger darf gemäß $§ 670$ $B G B$ auch Aufwendungen ersetzt verlangen, die zwar objektiv unnötig waren, die er aber für erforderlich halten durfte. Auf diese Weise vermindert $§ 670$ BGB die Gefahr, daß der Geschäftsbesorger, der über einen unkontrollierbaren wirtschaftlichen Ermessensspielraum verfügt, das Risiko nutzloser Aufwendungen zu Lasten einer optimalen Wahrung der wirtschaftlichen Interessen des Auftraggebers minimiert. Mit anderen Worten: Die Regelung des $\S 670$ BGB nimmt dem Geschäftsbesorger den Anreiz, an möglicherweise nutzlosen Aufwendungen, die er aus eigener Tasche tragen müßte, zu sparen und eine suboptimale Förderung der Interessen des Auftraggebers hinzunehmen; denn auch der entgeltlich tätige Geschäftsbesorger kann sichergehen, daß er selbst riskante Aufwendungen ersetzt erhält, sofern ihm nur kein Verschulden zur Last fällt. ${ }^{58)}$ Auf der gleichen Linie liegt $§ 664$ Abs. 1 Satz 2 BGB. Auch hier soll dem Geschäftsbesorger dadurch, daß er nur für culpa in eligendo haften muß, der Anreiz zur Vernachlässigung der Interessen des Auftraggebers genommen werden. Es soll die Gefahr eliminiert werden, daß der Beauftragte im Rahmen seines Ermessensspielraums zu Lasten des Geschäftsherrn (§670 BGB) den Auftrag kostspieliger ohne die Einschaltung von Substituten oder mit einem zwar mit Sicherheit solventen, aber unverhältnismäßig teueren Substituten ausführt, obwohl dies letztlich den wahren Interessen des Auftraggebers zuwiderläuft, der primär an niedrigen oder den Risiken optimal entsprechenden Kosten interessiert ist. ${ }^{59)}$

2.3.4 Als Fazit läßt sich mithin festhalten, daß die ratio des $\S 664$ Abs. 1 Satz 2 BGB aus heutiger Sicht in der Vermeidung von Interessenkonflikten, in der Motivierung des Geschäftsbesorgers zu optimaler Geschäftsbesorgung besteht.

\subsection{Voraussetzungen einer Analogie}

Die enge Verwandtschaft zwischen der ratio des $\S 664$ Abs. 1 Satz 2 BGB und der des $\S 670$ BGB, den $\S 675$ BGB ausdrücklich für entsprechend anwendbar erklärt, spricht eindringlich für eine analoge Anwendung des $\S 664$ Abs. 1 Satz 2 BGB im Rahmen des $\S 675$ BGB. Sowohl beim unentgeltlichen als auch beim entgeltlichen Geschäftsbesorgungsvertrag stehen dem Geschäftsherrn typischerweise Ermessensspielräume offen. Bei beiden Vertragsvarianten besteht daher normalerweise die Gefahr, daß der Geschäftsbesorger die Interessen des Geschäftsherrn vernachlässigen würde, wenn er verschuldensunabhängig Risiken des Geschäfts tragen müßte.

Aus der ratio des $\S 664$ Abs. 1 Satz 2 BGB ergibt sich jedoch zugleich, daß eine Analogie im Rahmen entgeltlicher Geschäftsbesorgungsverträge nur mit Einschränkungen möglich ist. Die von dem konkreten Ermessensspielraum unabhängige Regelung des $\S 664$ Abs. 1 Satz 2 BGB ist dadurch zu erklären, daß $\S 664$ Abs. 1

54) v. Gablenz (Fußn. 20), S. 133; Koller, Das Haftungsprivileg des Geschäftsbesorgers gem. $\S \S 664$ Abs. 1 Satz 2, 675 BGB, ZIP 1985, 1243, $1244 \mathrm{f}$.

55) Prot II, S. 378; vgl. aber zu §27 BGB: BGH, Urt. v. 14.12.1987,ZIP 1988, 706 $=$ EWiR $\S 27$ BGB 1/88, 427 (Reuter) =WM 1988, 531, 532.

56) Vgl. auch v. Gablenz (Fußn. 20), S. 87 ff; Koller, ZIP 1985, 1243, 1244.

57) Koller, ZIP 1985, 1243, 1244.

58) Koller, ZIP 1985, 1243, $1245 \mathrm{f}$.

59) Hierzu ausführlich: Koller, ZIP $1985,1243,1245 \mathrm{f}$. 
Satz 2 BGB unter dem Aspekt der Unentgeltlichkeit auch die Dienst-und Werkverträge ohne Geschäftsbesorgungscharakter erfassen kann. Der Gesetzgeber brauchte im Auftragsrecht nicht zu differenzieren, da die Regelung des $\S 664$ Abs. 1 Satz 2 BGB wegen der Unentgeltlichkeit des Auftrags selbst dort sachgerecht ist, wo im Einzelfall die Gefahr eines Interessenkonflikts undenkbar ist. Im Bereich entgeltlicher Geschäftsbesorgungsverträge ist ein derartiger Verzicht auf Differenzierung jedoch nicht möglich. Dort muß darauf abgestellt werden, ob im Einzelfall im Rahmen der Frage, ob ein Substitut und welcher Substitut eingeschaltet wird, ein Interessenkonflikt der geschilderten Art entstehen konnte. Nur wenn dies zu bejahen ist, ist eine Analogie zu $§ 664$ Abs. 1 Satz 2 BGB gerechtfertigt.

Demnach setzt eine Analogie zu $\S 664$ Abs. 1 Satz 2 BGB voraus, daß der Substitut in einem Bereich eingeschaltet wird, in dem dem Geschäftsbesorger ein wesentlicher Ermessensspielraum bei der Abwicklung der ihm obliegenden Aufgaben offensteht.

Ferner ist zu fordern, daß der Geschäftsbesorger den Substituten auf Rechnung des Auftraggebers ( $\$ 670$ BGB) einsetzt. Denn wenn der Geschäftsbesorger auf eigene Rechnung tätig wird, wird er ohnehin geneigt sein, um seinen Gewinn zu maximieren, nicht den besten, sondern den billigsten Substituten zu nehmen. Diese Tendenz würde noch verstärkt, wenn er für den Substituten nicht haften müßte. ${ }^{60)}$ So sieht auch $\S 413$ Abs. 1 HGB vor, daß der Spediteur, der auf eigene Rechnung arbeitet, ohne Rücksicht auf eigenes Verschulden für die von ihm eingeschalteten Frachtführer zu haften hat ( $\$ 431$ HGB). Die Kommission zur Beratung eines ADHGB hatte nämlich bei der Erörterung des Vorläufers des $\S 413$ Abs. 1 HGB hervorgehoben, daß dem Spediteur, der auf eigene Rechnung arbeite, „die Möglichkeit gegeben (werde), mit den wohlfeilsten Mittelspersonen zugleich auch die minder zuverlässigen auszuwählen “. ${ }^{61)} \mathrm{Ob}$ auch $\S 664$ Abs. 1 Satz 1 BGB im Rahmen des $\S 675$ BGB analog anzuwenden ist, kann hier offen bleiben. Es ergibt sich nämlich schon aus $\S 613$ Satz 1 BGB, daß die Bank nicht ohne zumindest konkludente Genehmigung einen Substituten einschalten darf. Wenn sie ungerechtfertigt Substituten einschaltet, haftet sie uneingeschränkt wegen positiver Forderungsverletzung, ohne sich auf $\S 664$ Abs. 1 Satz 2 BGB berufen zu können. ${ }^{62)}$

\section{Anwendung von $\S 664$ Abs. 1 Satz 2 BGB bei der Einschaltung von $Z$ wischenbanken}

Auf die Zuziehung von Zwischenbanken zur Durchführung einer Giroüberweisung könnte $\S 664$ Abs. 1 Satz 2 BGB demnach nur dann analog angewendet werden, wenn die Erstbank dabei auf fremde Rechnung handeln würde und die Gefahr eines Mißbrauchs ihres Ermessens bestehen würde.

\subsection{Handeln auf fremde Rechnung}

Wenn der Erstbank von der Zwischenbank Kosten und Gebühren in Rechnung gestellt werden, wälzt sie sie über $\S \S 670,675$ BGB direkt auf den Kunden ab, handelt also auf dessen Rechnung. In Frage stehen damit nur ,indirekte“ Vergütungen der Zwischenbank. Das sind zunächst ihre Wertstellungsgewinne, die sie insofern auf Kosten der Erstbank zieht, als sie sie dieser eigentlich nach $\S \S 667,675$ BGB herauszugeben hätte, während die Erstbank dem Auftraggeber gegenüber aufgrund des Girovertrags von vornherein von dieser Herausgabepflicht entbunden ist. Doch die Beträge, auf die die Erstbank so zugunsten der Zwischenbank verzichtet, sind im Einzelfall so gering, daßs ihre Berechnung viel zu aufwendig wäre und sie die Auswahlentscheidung der Erstbank (zugunsten einer Zwischenbank, die die Wertstellungsgewinne herausgibt) wohl kaum beeinflussen werden. Gleiches gilt für die zweite Art „indirekter“ Vergütung, nämlich die Kosten, die der Erstbank dadurch entstehen, daß sie ihrerseits unentgeltlich für die Zwischenbank tätig wird. Außerdem wird die Erstbank an einer solchen Tätigkeit häufig sogar interessiert sein, weil sie auf diese Weise Wertstellungsgewinne erzielen kann.

Weit eher, als daß diese Faktoren die Auswahlentscheidung beeinflussen, ist dagegen anzunehmen, daß die Erstbank bei Nichtanwendung des $\S 664$ Abs. 1 Satz 2 BGB unter Vernachlässigung der Kundeninteressen den für sie haftungsmäßig günstigsten Überweisungsweg einschlägt. Die Tatsache, daß die Erstbank in kleinerem Umfang auf eigene Rechnung tätig wird, steht daher der analogen Anwendung des $\S 664$ Abs. 1 Satz 2 BGB nicht entgegen.

Der Gefahr, daß eine Zwischenbank nur deshalb ausgewählt wird, weil sie in Form von Kompensationsgeschäften ihrerseits die Erstbank als Zwischenbank beauftragt und ihr so Wertstellungsgewinne ermöglicht, kann durch die Anwendung oder Nichtanwendung von $\S 664$ Abs. 1 Satz 2 BGB allerdings nicht begegnet werden, ein derartiger Mißbrauch des Leistungsermessens muß mit Hilfe der Haftung für culpa in eligendo sanktioniert werden.

\subsection{Ermessensspielraum der Erstbank}

3.2.1 Köndgen verneint die Anwendbarkeit des $§ 664$ Abs. 1 Satz 2 BGB mit dem Argument, die Zwischenbank verfüge nicht über jenes Leistungsermessen, das den Vertragstyp „Geschäftsbesorgung" kennzeichne. Schon im Verhältnis Kunde - Erstbank sei das Geschäftsbesorgungselement nicht sonderlich ausgeprägt; bei der Tätigkeit der Zwischenbank sei davon fast nichts mehr übrig, weil den Zwischenbanken nicht wesentliche Teile der Geschäftsbesorgung übertragen würden und ein großer Teil der Arbeit maschinell erledigt werde. Das Leistungsermessen der Zwischenbank beschränke sich auf die Auswahl der nächsten Zwischenbank. ${ }^{63)}$

Köndgens Ansicht kann nicht gefolgt werden, selbst wenn man unterstellt, daß die Zwischenbank keinen eigenen Ermessensspielraum hat. Denn im Rahmen von $\S 664$ Abs. 1 Satz 2 BGB kommt es nicht darauf an, ob der Substitut einen Ermessensspielraum hat, sondern darauf, ob ihn der eigentliche Geschäftsbesorger besitzt, der von Interessenkonflikten entlastet werden muß.

60) Vgl. Koller, ZIP 1985, 1243, 1246 ff.

61) v. Lutz, Protokolle der Kommission zur Berathung eines ADHGB (1858), S. 777, 1223; vgl. auch Koller, Die Verweisung der $§ \S 412,413$ HGB auf die CMR VersR 1987, 1058, 1061 .

62) Mot II, S. 534; MünchKomm-Seiler(Fußn. 25), §664 Rz. 10; Steffen, in: BGB RGRK (Fußn. 38), §664 Rz. 9; Soergel/Mübl (Fußn. 25), §664 Rz. 3; Staudinger/ Wittmann (Fußn. 25), §664 Rz.3.

63) Köndgen (Fußn. 4), S. 149 f. 
3.2.2 Entscheidend ist mithin, ob der Erstbank bei der Durchführung der Überweisung ein Ermessensspielraum offenstand, in dessen Ausfüllung die Zwischenbank eingeschaltet wurde. Dies ist eine Frage des Einzelfalls, in der Regel dürfte die Erstbank jedoch einen ganz erheblichen Ermessensspielraum besitzen: Im Auslandsverkehr ist dies offensichtlich, da der Erstbank häufig keine eigenen Filialen in dem betreffenden Land zur Verfügung stehen und sie deshalb geeignete Zwischenbanken auswählen muß, die eine optimale Ausführung der Überweisung erwarten lassen. Auch im Inlandsverkehr gibt es jedoch eine Vielzahl möglicher Überweisungswege: Die Erstbank kann, wenn sie mit der Empfängerbank in Kontoverbindung steht, direkt an sie überweisen, sie kann die Überweisung im eigenen Netz bis zu einer Filiale leiten, die in Geschäftsverbindung mit der Empfängerbank oder einer ihrer Filialen steht, oder sie kann die Landeszentralbank, eine andere Zwischenbank oder das Postgironetz einschalten. Vor allem bei Eil-Überweisungen, die nach 11.30 Uhr von den Landeszentralbanken nicht mehr ausgeführt werden, sind all diese Möglichkeiten gebräuchlich.

Im Einzelfall kann der Ermessensspielraum freilich auf Null reduziert sein, etwa wenn einer der zur Auswahl stehenden Wege gegenüber den anderen beträchtliche Vorteile bietet (z.B. in bezug auf die Schnelligkeit der Überweisung). Die Erstbank haftet dann für das Verschulden der Zwischenbanken nach §278 BGB.

Sofern der Erstbank aber - wie dies normalerweise der Fall ist ein Entscheidungsspielraum offensteht, gebietet es die ratio von $\S 664$ Abs. 1 Satz 2 BGB - nämlich den Kunden von der Gefahr eines nicht nachweisbaren Mißbrauchs des Leistungsermessens der Bank zu entlasten - die Erstbank nur für eigenes Verschulden (culpa in eligendo vel instruendo) haften zu lassen. Auf diese Weise wird im Interesse des Kunden verhindert, daß die Erstbank allzusehr ihre eigenen Interessen in den Vordergrund rückt, daß sie z.B. mit dem Argument der Kostengünstigkeit Überweisungswege bevorzugt, die zwar wesentlich langsamer sind, sie aber weniger dem Risiko einer Haftung für schuldhaftes Verhalten einer Zwischenbank aussetzen.

3.2.3 Man kann sogar einen Schritt weiter gehen und behaupten, daß die Existenz eines Ermessensspielraums für sich allein nicht die Anwendung des $\S 664$ Abs. 1 Satz 2 BGB auf entgeltliche Geschäftsbesorgungsverträge rechtfertigen könne. Es müsse vielmehr die konkrete Gefahr eines Ermessensmißbrauchs durch die Erstbank bestehen, die nur bei konfligierenden Interessen von ihr und dem Kunden möglich sei.

Ein Ermessensmißbrauch ist typischerweise zu befürchten, wenn die Erstbank den Überweisungsauftrag auch ohne Einschaltung einer Zwischenbank ausführen kann. Hier könnte sie nämlich geneigt sein, keine Zwischenbank zuzuziehen, um so das Fehlerrisiko „in der Hand zu behalten“ und sich im Schadensfall nicht mit anderen Banken auseinandersetzen zu müssen, obwohl die Überweisung durch Einschaltung einer sicheren und zuverlässigen Zwischenbank schneller oder kostengünstiger auszuführen wäre - die Gefahr eines nicht nachweisbaren Mißbrauchs des Leistungsermessens ist erheblich. Von ihr kann der Kunde nur durch die Anwendung des $§ 664$ Abs. 1 Satz 2 BGB, der die Haftung der Erstbank auf eigenes Verschulden (culpa in eligendo vel instruendo) beschränkt, entlastet werden.
Anders liegt es dagegen, wenn die Erstbank den Auftrag pflichtgemäß nicht ohne Einschaltung einer Zwischenbank ausführen kann und sich ihr Ermessen deshalb auf die Auswahl der Zwischenbank beschränkt. Die Anwendung von $§ 664$ Abs. 1 Satz 2 BGB kann den Auftraggeber vor der Berücksichtigung von zwei eigenen Interessen der Erstbank bei dieser Auswahlentscheidung schützen: es gar nicht zu einem Fehler kommen zu lassen und daher nur eine absolut zuverlässige Zwischenbank zu nehmen sowie im Fall einer Haftung für die Zwischenbank bei dieser mit Sicherheit Regreß nehmen zu können und daher nur eine absolut solvente Zwischenbank auszuwählen.

$\mathrm{Da}$ nur eine absolut zuverlässige und solvente Zwischenbank beauftragt wird, liegt aber typischerweise auch im Interesse des Kunden, da so eine ordnungsgemäße Abwicklung der Überweisung gewährleistet und die Gefahr, bei Insolvenz der Zwischenbank den Überweisungsbetrag zu verlieren, minimiert wird. Eine „schlampig“ oder „unsicher" erscheinende Zwischenbank darf die Erstbank daher schon im Interesse des Kunden nicht einschalten. Ein Interessenkonflikt der Erstbank ist somit nur insofern möglich, als sie eventuell bei der Auswahl unter mehreren als hinreichend zuverlässig und als mit Sicherheit solvent bekannten Banken die absolut beste wählen wird, auch wenn andere billiger oder schneller arbeiten.

Man könnte weiter argumentieren, die Gefahr, daß die Erstbank diese geringen Unterschiede an Zuverlässigkeit und Sicherheit entgegen den Kundeninteressen zum bestimmenden Faktor ihrer Auswahlentscheidung machen werde, erscheine sehr gering. Da nun die analoge Anwendung des $\S 664$ Abs. 1 Satz 2 BGB bei entgeltlicher Geschäftsbesorgung allein den Schutz des Geschäftsherrn bezwecke, sei sie dann nicht gerechtfertigt, wenn sie sich für diesen im Ergebnis nachteilig auswirken würde. So liege es hier: Die Ausschaltung der geringen Gefahr eines Ermessensmißbrauchs durch die Erstbank „bezahle“ der Auftraggeber mit deren stark eingeschränkter Haftung. Bei Verschulden einer Zwischenbank könne er sich nur noch an diese halten, müsse das Risiko ihrer Insolvenz tragen und könne Schadensersatzansprüche gegen sie nur unter erheblichen Schwierigkeiten durchsetzen. Der geringe „Gewinn“, den die Ausschaltung von Interessenkonflikten auf Seiten der Erstbank hier dem Kunden bringe, könne diese Nachteile nicht kompensieren.

Dieser Lösungsansatz macht es erforderlich, danach zu differenzieren, wie groß im Einzelfall die Gefahr eines Ermessensmißbrauchs war. Auf diese Weise gelangt man zwar zu Lösungen, die der ratio des $\S 664$ Abs. 1 Satz 2 BGB am nächsten kommen. Man hat jedoch auch mit erheblichen Praktikabilitätsproblemen zu kämpfen, denn in jedem Streitfall müßte dargelegt und bewiesen werden, ob die Gefahr eines Ermessensmißbrauchs mehr oder weniger gravierend war. In dieser Situation spricht die Rechtssicherheit für eine stärker generalisierende Analogie zu $\S 664$ Abs. 1 Satz 2 BGB, die nur die Existenz eines Ermessensspielraums voraussetzt. ${ }^{64)}$

64) Der Mitautor Faust vertritt dagegen - methodisch gleichermaßen vertretbar - die Auffassung, die Rechtssicherheit könne nicht so stark ins Gewicht fallen, daß die analoge Anwendung von $§ 664$ Abs. 1 Satz 2 BGB schon beider Existenz eines Ermessensspielraums des Geschäftsbesorgers gerechtfertigt sei. Aus der ratio des $\S 664$ Abs. 1 Satz 2 BGB heraus sei vielmehrals Haftungsvoraussetzung die Gefahr eines Ermessensmißbrauchs zu fordern, die freilich ihrerseits, um überzogene Beweisanforderungen zu vermeiden, typisiert bestimmt werden 


\subsection{Zwischenergebnis}

In aller Regel ist also $§ 664$ Abs. 1 Satz 2 BGB auf die Einschaltung von Zwischenbanken analog anwendbar, da die Erstbank dabei normalerweise einen Ermessensspielraum hat und nicht auf eigene Rechnung handelt.

\section{Tatbestandsvoraussetzungen von $§ 664$ Abs. 1 Satz 2 BGB}

$\S 664$ Abs. 1 Satz 2 BGB setzt voraus, daß eine Substitution vorliegt, also die Übertragung des Auftrags an einen Dritten $(\$ 664$ Abs. 1 Satz 1 BGB) im Gegensatz zur Zuziehung eines Gehilfen ( $\$ 664$ Abs. 1 Satz 3 BGB), und daß diese Substitution gestattet ist.

\section{1 Übertragung des Auftrags an einen Dritten}

Das Reichsgericht nahm an, Substitution bedeute eine vollständige Übertragung der Geschäftsbesorgungspflicht; diese sei immer dann gegeben, wenn einem Dritten der Auftrag insgesamt oder teilweise dergestalt übertragen werde, daß er seinem Auftraggeber selbständig wie ein Geschäftsbesorger dem Geschäftsherrn gegenüberstehe. ${ }^{65)}$ Der Unterschied zur neueren Lehre, die ganz auf die Selbständigkeit des Dritten abstellt, ${ }^{66)}$ liegt nur in der Formulierung: Entscheidend ist, daß bei der (deshalb im Zweifel verbotenen) Substitution der Beauftragte, in den der Auftraggeber sein besonderes Vertrauen gesetzt hat, nicht „Herr des Geschehens“ bleibt, sondern die Tätigkeit des Dritten seinem Einfluß und Kontrollbereich weitgehend entzogen ist. Die Einschaltung einer Zwischenbank erfüllt diese Voraussetzungen nicht, wenn Erst- und Zwischenbank lediglich verschiedene Filialen des gleichen Kreditinstituts sind. Denn obwohl die einzelnen Filialen eine gewisse Selbständigkeit haben, sind sie Teil der einheitlichen Organisationsstruktur des Instituts, das sie zentral überwacht und steuert. Ansonsten liegt in der Regel Substitution vor. Eine Ausnahme wäre nur zu machen, wenn das erstbeauftragte Institut die Zwischenbank aufgrund vertraglicher Beziehungen so beherrscht, daß es jederzeit in ihren Betriebsablauf eingreifen kann; die Erteilung detaillierter Weisungen in bezug auf die Behandlung des Überweisungsauftrags genügt nicht.

\subsection{Gestattung der Substitution}

4.2.1 Nach $\S 613$ Satz 1 BGB und auch nach $\S 664$ Abs. 1 Satz 1 BGB, wenn man ihn analog anwendet, muß die Erstbank die Überweisung „im Zweifel“ ausschließlich mit Hilfe ihrer eigenen Organisation, ohne Einschaltung von Zwischenbanken, erledigen. Es fragt sich daher, ob entgegen diesen gesetzlichen Auslegungsregeln der konkrete Girovertrag die Substitution gestattet.

4.2.2 Der Bankkunde wird kaum jemals einen realen Willen des Inhalts bilden, daß die Substitution erlaubt sein soll. Er wird nämlich in aller Regel nicht wissen, daß die Einschaltung von Zwischenbanken sinnvoll sein kann und daß sie von seiner Erlaubnis abhängt.

4.2.3 Die Erlaubnis zur Substitution kann sich jedoch durch Auslegung des Girovertrags nach $\S 157$ BGB ergeben, ${ }^{67}$ wenn ein redlicher Kunde aus der Sicht der Erstbank mit der Beauftragung einer Zwischenbank einverstanden wäre. Die Bank muß sich also in erster Linie an den ihr erkennbaren Interessen ihres Kunden orientieren. Macht er z. B. deutlich, daß er nur „seiner Bank" vertraue, so gestattet er keine Substitution. In der Regel werden Kunden aber ihren Willen bezüglich einer Substitution nicht offenlegen. In dieser Situation ist die Erstbank nicht darauf angewiesen, in jedem Einzelfall die Vorstellungen des Kontoinhabers zu erforschen. Sie kann sich am hypothetischen Willen eines Durchschnittskunden orientieren. Für den redlichen Durchschnittskunden werden bei der Entscheidung der Frage, ob er mit einer Substitution einverstanden ist, im wesentlichen drei Faktoren eine Rolle spielen: die Solvenz der Zwischenbank, die Schnelligkeit und die Wirtschaftlichkeit der Überweisungsausführung.

Wird eine dubiose Zwischenbank eingeschaltet, läuft der Kunde nicht nur Gefahr, etwaige Schadensersatzansprüche gegen diese nicht durchsetzen zu können, sondern auch, wegen $§ 670$ BGB, den Überweisungsbetrag zu verlieren. Der durchschnittliche Kunde wird daher besonderes Gewicht auf eine möglichst sichere Überweisungsausführung legen. Das heißt nicht, daß er wegen der nach $\S 664$ Abs. 1 Satz 2 BGB nur beschränkten Haftung der Erstbank die Einschaltung von Zwischenbanken generell ablehnt. Man wird jedoch annehmen müssen, daß er mit der Einschaltung einer Zwischenbank nicht einverstanden ist, wenn sich aus der Sicht eines objektiven außenstehenden Beobachters auch nur der geringste Zweifel an ihrer Solvenz regt. Dies gilt selbst dann, wenn eine Überweisung ohne Zuziehung von Zwischenbanken zweifelhafter Solvenz nicht ausgeführt werden könnte; in diesem Fall muß die Erstbank den Kunden informieren und ihrer Entscheidung dann seinen realen Willen zugrunde legen.

Bankkunden, die einen Überweisungsauftrag erteilen, sind typischerweise an einer möglichst schnellen Gutschrift zugunsten des Empfängers interessiert. Dies ist dort evident, wo es um die Begleichung von Bringschulden (z. B. Steuerschulden, $\$ 224$ Abs. 2 Nr. 2 AO) geht. Aber auch wenn die Schuld des Überweisenden eine qualifizierte Schickschuld ( $\$ 270$ BGB) ist und somit der Empfänger das Verzögerungsrisiko trägt, wird dem Kunden häufig an einem schnellen Lauf der Überweisung gelegen sein, etwa weil er die Gegenleistung erst nach Gutschrift erhält, weil von der schnellen Gutschrift der Abzug eines Skontos abhängt oder weil er seine Geschäftsbeziehungen pflegen will. Die Bank kann und muß daher davon ausgehen, daß der Kunde an möglichst schneller Erledigung der Überweisung interessiert und grundsätzlich auch bereit ist, dafür die Einschaltung einer Zwischenbank hinzunehmen.

könne. Was die Durchführung einer Giroüberweisung betreffe, sei sie, wie darge legt, typischerweise dann gegeben, wenn die Erstbank innerhalb ihres Ermes sensspielraums den Überweisungsauftrag sowohl ohne als auch mit Einschal tung von Zwischenbanken ausführen könne. Müßten dagegen notwendigerweise $Z$ wischenbanken eingeschaltet werden und beschränke sich das Ermessen der Erstbank auf deren Auswahl, könne die Gefahr eines Ermessensmißbrauchs vernachlässigt werden. Nurim ersten Fall sei daher die analoge Anwendung von $\S 664$ Abs. 1 Satz 2 BGB gerechtfertigt.

65) RG, Urt. v. 2.3.1912, RGZ 78, 310, 312, 314; ähnlich MünchKomm-Hanau (Fußn. 25), §278 Rz. 16; Steffen, in: BGB-RGRK(Fußn. 38), §278 Rz. 25; Soergel Mübl (Fußn. 25), §664 Rz.2

66) MünchKomm-Seiler (Fußn. 25), §664 Rz.4; v. Gablenz (Fußn. 20), S. $124 \mathrm{ff}$; Koller, ZIP 1985, 1243, 1247

67) MünchKomm-Seiler (Fußn. 25), §664 Rz. 5; Soergel/Mübl (Fußn. 25), §664 Rz. 3; Staudinger/Wittmann (Fußn. 25), §664 Rz. 5. 
Dem Kunden wird ferner an einer kostengünstigen Abwicklung des Überweisungsauftrags gelegen sein. Dieses Interesse erschöpft sich nicht darin, daß die im Einzelfall berechneten Überweisungsgebühren niedrig sind. Dem wirtschaftlich denkenden Girokunden wird darüber hinaus auch an einer Minimierung der allgemeinen Betriebskosten der Bank im Giroverkehr gelegen sein. Eine Erhöhung dieser Kosten müßte ihn nämlich zwangsläufig treffen, indem sich die Bedingungen, zu denen sein Konto geführt wird (Zinsen, Gebühren, Wertstellung, "Service“), verschlechtern. Umgekehrt führen niedrige Kosten unter den Bedingungen eines funktionierenden Marktes zu Vorteilen für den Kunden. Daher werden sich verständige Kunden nicht gegen die Einschaltung von Zwischenbanken sperren, wenn dies per Saldo eine kostengünstigere Abwicklung der Überweisung erlaubt.

4.2.4 Der Durchschnittskunde wird also mit der Einschaltung einer Zwischenbank einverstanden sein, wenn sie seinem Interesse an einer schnellen und wirtschaftlichen ${ }^{68)}$ Durchführung der Überweisung dient und an der Solvenz der Zwischenbank aus der Sicht eines ordentlichen Bankiers keinerlei Zweifel besteht. ${ }^{69)}$ Die Beweislast für die Gestattung der Substitution trägt die Erstbank. ${ }^{70)}$

\section{Haftung für culpa in eligendo}

Die Erstbank haftet nach allgemeinen Rechtsgrundsätzen, die durch $\S 664$ Abs. 1 Satz 2 BGB bestätigt werden, im Fall einer erlaubten Substitution, falls sie eine ungeeignete Zwischenbank ausgewählt hat. Auch bei der Auswahl der Zwischenbank ist sie gehalten, sich an den Kundeninteressen zu orientieren. Macht z.B. der Auftraggeber deutlich, daß ihm an einer möglichst schnellen Durchführung der Überweisung gelegen ist, so muß primär diesem Interesse Rechnung getragen werden. Zur Erforschung der Kundenpräferenzen, etwa anhand der Vermerke auf dem Überweisungsträger, ist die Erstbank allerdings nicht verpflichtet. Wenn ihr der Kunde nicht atypische Interessen offenbart, kann sie davon ausgehen, daß er eine möglichst schnelle und möglichst wirtschaftliche Ausführung der Überweisung wünscht.

Konfligieren diese Interessen, hat die Erstbank bei der Auswahl des günstigsten Überweisungsweges einen geschäftsbesorgungstypischen Ermessensspielraum, innerhalb dessen sie die Wahl optimieren muß. Diese Optimierung kann, da es um den „hypothetischen Durchschnittskunden“ geht, generell-typisch vorgenommen und daher zum Bestandteil von Computerprogrammen gemacht werden. Der der Bank offenstehende Ermessensspielraum ist jedoch überschritten, wenn sie eine Zwischenbank einschaltet, deren Solvenz nicht über allen Zweifel erhaben ist, oder wenn sie unverhältnismäßig viel Gewicht auf Schnelligkeit oder Wirtschaftlichkeit legt. Erst recht handelt die Erstbank bei der Auswahl der Zwischenbank schuldhaft, wenn sie sich von eigenen Gewinninteressen leiten läßt. So muß ihr Ermessensmißbrauch vorgeworfen werden, falls sie in Erwartung von Aufträgen einer Zwischenbank oder zur Belohnung früherer Aufträge selbst Überweisungen über diese Zwischenbank leitet, um auf diese Weise die eigenen Wertstellungsgewinne bzw. die Wertstellungsgewinne einer Gruppe zu erhöhen. Daß sie die Auswahl der Zwischenbank im Einklang mit ihren Sorgfaltspflichten getroffen hat, muß die Erstbank beweisen.

\section{Haftung im Licht der AGB-Banken}

\section{Nr. 9 AGB-Banken}

Die Erstbank könnte sich, wenn sie auch in ihrem eigenen Interesse (und damit nach dispositivem Recht unzulässigerweise) substituiert, möglicherweise auf Nr. 9 Satz 1 AGB-Banken berufen.

Diese Klausel wiederholt entgegen $\mathrm{Kümpel}^{71)}$ nicht nur $\S 664$ Abs. 1 Satz 1 BGB, denn im Gegensatz zu dieser Vorschrift gestattet sie die Substitution generell ${ }^{72)}$.

Nr.9 Satz 1 AGB-Banken verstößt gegen $\$ 9$ AGBG. Selbst wenn man die Auffassung vertritt, daß die Berücksichtigung der Bankeninteressen in Nr. 9 AGB-Banken grundsätzlich mit $\S 315$ Abs. 3 BGB im Einklang steht, ${ }^{73)}$ widerspricht die Klausel dem Grundgedanken des Geschäftsbesorgungsrechts. Für Geschäftsbesorgungsverträge ist typisch, daß der Beauftragte sich ausschließlich vom Interesse des Auftraggebers leiten lassen muß. Nur dies rechtfertigt es, daß der Auftraggeber das Risiko objektiv nutzloser Aufwendungen trägt ( $\$ 670$ BGB) und den Auftragnehmer nicht für schuldhaftes Verhalten eines Substituten haftbar machen kann. Wenn nun die Bank auch in ihrem eigenen Interesse Dritte einschalten darf, wird das Haftungsprivileg des $\S 664$ Abs. 1 Satz 2 BGB zu einem Anreiz, dies in möglichst großem Umfang zu tun und so die eigene Haftung weitestgehend zu beschränken. Nr. 9 Satz 1 AGB-Banken ist daher wegen Verstoßes gegen $§ 9$ AGBG unwirksam. ${ }^{74 \text { ) }}$

\section{Nr. 25 Abs. 1 AGB-Banken}

Kann sich die Erstbank nicht auf $\$ 664$ Abs. 1 Satz 2 BGB berufen, so stellt sich die Frage, ob sie Nr. 25 Abs. 1 AGB-Banken ins Feld führen kann. Diese Klausel wird allgemein als wirksam angesehen, da sie in Übereinstimmung mit $§ 11$ Nr. 7 AGBG steht und die Erstbank häufig auf die Einschaltung von Zwischenban-

68) Spricht der Gesichtspunkt der Schnelligkeit gegen die Einschaltung von Zwischenbanken, der Gesichtspunkt der Wirtschaftlichkeit aber dafür oder umgekehrt (was in der Praxis selten der Fall sein wird), gilt das nachstehend bezüglich der culpa in eligendo Gesagte.

69) Das gilt-entgegen v. Gablenz (Fußn. 20), S. 228 -auch bei der Filialüberweisung.

70) BGH, Urt. v. 14.11.1951, LM BGB $§ 664$ Nr. 1; MünchKomm-Seiler (Fußn.25), §664 Rz.9; Soergel/Mühl (Fußn.25), §664 Rz.3; Staudinger/Wittmann (Fußn. 25), §664 Rz. 1; Steffen, in: BGB-RGRK (Fußn. 38), §664 Rz. 13.

71) Kümpel, WM 1977, 694, 699; ebenso MünchKomm-Seiler (Fußn. 25), §664 Rz. 17; und wohl auch Graf v. Westphalen, in: Loewe/Graf v. Westphalen/Trinkner, AGBG, Bd.III, 2. Aufl., 34.1 Rz.28.

72) Brandner, in: Ulmer/Brandner/Hensen, AGBG, 5.Aufl., Anh. §§9-11 Rz.159; Canaris, Bankvertragsrecht, 2.Aufl., 1981, Rz.2586; v. Gablenz (Fußn. 20), S. 186 ff; Lwowski, Das AGB-Gesetz und die AGB der Banken, Die Bank 1978, 123, 129, Fußn.27; Schlenke, Allgemeine Geschäftsbedingungen der Banken und AGB-Gesetz, 1984, S.35f.

73) Aus diesem Grund halten die Klausel für unbedenklich: v. Gablenz (Fußn. 20), S.200ff; Lwowski, Die Bank 1978, 123.

74) Brandner(Fußn. 72), Anh. §§9-11 Rz. 159; Horn, in: Wolf/Horn/Lindacher, AGBG, 2. Aufl., §23 Rz.643; Baumbach/Duden/Hopl, HGB, 27. Aufl., Nr. 9 AGB-Banken Anm. 1; Canaris (Fußn. 72), Rz. 2586; Heinrichs, Freizeichnung im Bankgeschäft, in: Köndgen, Neue Entwicklungen im Bankhaftungsrecht, 1987, S. 115, 127 f; Koller, ZIP 1985, 1243, 1249; Schlenke (Fußn. 72), S. 36; im Ergebnis auch Staudinger/Schlosser (Fußn.25), §10 Nr. 3 AGBG Rz.21. 
ken angewiesen ist, diese aber nur schwer kontrollieren kann. ${ }^{75)}$ Canaris will allerdings die Klausel restriktiv dahingehend auslegen, daß sie sich nicht auf Aufgaben beziehe, die traditionsgemäß eigenen Mitarbeitern der Bank obliegen. ${ }^{76)}$

Diese Einschränkung zeigt, daß es letztlich auf die für die Gültigkeit der Klausel sprechenden Argumente der erschwerten Kontrollierbarkeit und Angewiesenheit doch nicht so sehr ankommt. §278 BGB liegt die Wertung zugrunde, daß es Sache des Schuldners ist, zu entscheiden, wie er seine Aufgaben erfüllt: persönlich, mit weisungsabhängigen Arbeitnehmern oder mit selbständigen Subunternehmern. Er braucht deshalb auch auf Sicherheitsinteressen seiner Gläubiger keine Rücksicht zu nehmen, muß zum Ausgleich aber gemäß §278 BGB für die mit der Einschaltung Dritterverbundenen Fehlerrisiken einstehen. Dieser innere Zusammenhang kann zerstört werden, wenn sich der Schuldner für einfaches Verschulden seiner Erfüllungsgehilfen gänzlich freizeichnet und dem Gläubiger das Risiko der Insolvenz des Schädigers zuschiebt. Eine Freizeichnung für leichte Fahrlässigkeit von Erfüllungsgehilfen kann daher wegen VerstoBes gegen $§ 9$ Abs. 1, Abs. 2 Nr. 2 AGBG unwirksam sein. ${ }^{77)}$ Dies wird allgemein bei sogenannten „Kardinalpflichten“ angenommen. Welche Pflichten Kardinalpflichten sind, soll sich aus der Natur des Vertrages ${ }^{78)}$ oder einem entsprechenden besonderen Vertrauenstatbestand ${ }^{79)}$ ergeben. Daß eine Bank für ihre Kunden Überweisungen vornimmt, ist eine ihrer zentralen Aufgaben und entspricht ihrer „Berufsrolle“, auf deren ordnungsgemäße Ausfüllung der Kunde naturgemäß besonders vertraut. ${ }^{80)}$ Da die Bank für die Ausführung von Überweisungen typischerweise selbständige Erfüllungsgehilfen einschaltet, würde ihre girovertragliche Pflicht zu sorgfältiger Erledigung von Überweisungen bei einer Freizeichnung für leichte Fahrlässigkeit von selbständigen Erfüllungsgehilfen großenteils ausgehöhlt, zumal grobes Verschulden einer Zwischenbank nur selten nachzuweisen sein wird. Nr.25 Abs. 12 . Halbs. AGB-Banken verstößt daher, zumindest was die Pflicht zu sorgfältiger Erledigung von Überweisungen anbelangt, gegen $\S 9$ Abs. 1 , Abs. 2 Nr. 2 AGBG und ist deshalb unwirksam. Will die Erstbank nicht haften, muß sie eben ihre Verpflichtung auf die Weiterleitung der Überweisung beschränken.

\section{Zusammenfassung}

(1) Möglich sind folgende Fallgestaltungen:

- Der Kunde erteilt Weisung, den Überweisungsauftrag durch Einschaltung eines bestimmten Dritten auszuführen.

Im Normalfall beschränkt sich dann die Verpflichtung der Bank auf die ordnungsgemäße Einschaltung dieses Dritten. Die Gründe für eine „weite“ Auslegung der Verpflichtung der Erstbank greifen in diesem Fall nicht ein: der Kunde ist über die
Modalitäten des Überweisungsverkehrs informiert und kann billigerweise nicht verlangen, daß die Erstbank das Risiko der Insolvenz eines Dritten trägt, auf dessen Auswahl sie keinerlei Einfluß hatte.

- Die Ausführung der Überweisung ist nur durch Einschaltung eines ganz bestimmten Dritten möglich, ohne daß der Erstbank eine culpa in eligendo zur Last fällt.

Da die Erstbank keinen Entscheidungsspielraum hat, liegen die Voraussetzungen für eine analoge Anwendung von $\S 664$ Abs. 1 Satz 2 BGB nicht vor. Die Erstbank haftet damit für die Zwischenbank nach $\S 278$ BGB. Will sie dieses Risiko nicht übernehmen, muß sie mit dem Kunden eine Sondervereinbarung treffen.

- Es gibt mehrere vertretbare Wege zur Ausführung der Überweisung; für den Kunden ist die Einschaltung eines selbständigen Dritten am günstigsten.

Hier liegt ein Fall zulässiger Substitution vor; nach $§ 664$ Abs. 1 Satz 2 BGB haftet die Erstbank nicht für die eingeschaltete Zwischenbank, sondern nur für eigenes Verschulden bei deren $\mathrm{Zu}$ ziehung (culpa in eligendo vel instruendo).

- Es gibt mehrere vertretbare Wege zur Ausführung der Überweisung; für den Kunden ist die Durchführung ohne Einschaltung einer Zwischenbank am günstigsten.

Die Erstbank darf keine Zwischenbank zuziehen. Tut sie es dennoch, haftet sie aus positiver Forderungsverletzung für jeden Schaden, für den die Einschaltung der Zwischenbank kausal war.

(2) Die Erstbank darf nur solche Zwischenbanken beauftragen, an deren Solvenz nicht der geringste Zweifel besteht. Unter mehreren derartigen Zwischenbanken hat sie diejenige auszuwählen, die den Interessen des Kunden an einer schnellen und wirtschaftlichen Überweisung am besten Rechnung zu tragen vermag. Soweit es um die Optimierung der möglicherweise entgegengesetzten Ziele „Schnelligkeit" und „Wirtschaftlichkeit“ geht, steht der Erstbank ein Ermessensspielraum zu.

75) Brandner (Fußn. 72), Anh. \$§9-11 Rz. 157; Horn (Fußn. 74), §23 Rz.635; Graf v. Westphalen (Fußn. 71), 34.1, Rz. 83; Baumbach/Duden/Hopt (Fußn. 74), Nr. 25 AGB-Banken Anm. 1; Schlenke (Fußn. 72), S. 34

76) Canaris (Fußn. 72), Rz.2717.

77) BGH, Urt. v. 21.12.1987, ZIP 1988, 306 =EWiR §9 AGBG 5/88, 319 (Rüm ker) $=$ WM 1988, 246, 248; Hensen, in: Ulmer/Brandner/Hensen (Fußn. 72), §11 Nr. 7 Rz. 23; Heinrichs (Fußn. 74), S. 129 f.

78) Hensen (Fußn. 77), §11 Nr. 7 Rz. 25

79) Graf v. Westphalen in: Loewe/Graf v. Westphalen/Trinkner, AGBG, Bd.II, 2. Aufl., §11 Nr.7 Rz.50; Wolf (Fußn. 74), §11 Nr.7 Rz.28; im Ergebnis wohl auch Staudinger/Schlosser (Fußn. 25), §11 Nr. 7 AGBG Rz. 37

80) Vgl. BGH, Urt. v. 21.12.1987, ZIP 1988, 360, 362 f; Koller, Die Wirksamkeit formularmäßiger Haftungsfreizeichnungsklauseln zwischen Schadensausgleich und Schadensprävention, ZIP 1986, 1089, 1099 m.w. N. 